JOURNAL OF THE AMERICAN MATHEMATICAL SOCIETY

Volume 14, Number 2, Pages 399-428

S 0894-0347(01)00358-7

Article electronically published on January 18, 2001

\title{
THE NUMBER OF REIDEMEISTER MOVES NEEDED FOR UNKNOTTING
}

\author{
JOEL HASS AND JEFFREY C. LAGARIAS
}

\section{INTRODUCTION}

A knot is an embedding of a circle $S^{1}$ in a 3-manifold $M$, usually taken to be $\mathbb{R}^{3}$ or $S^{3}$. In the 1920's Alexander and Briggs [2, §4] and Reidemeister 23] observed that questions about ambient isotopy of polygonal knots in $\mathbb{R}^{3}$ can be reduced to combinatorial questions about knot diagrams. These are labeled planar graphs with overcrossings and undercrossings marked, representing a projection of the knot onto a plane. They showed that any ambient isotopy of a polygonal knot can be achieved by a finite sequence of piecewise-linear moves which slide the knot across a single triangle, which are called elementary moves (or $\Delta$-moves). They also showed that two knots were ambient isotopic if and only if their knot diagrams were equivalent under a finite sequence of local combinatorial changes, now called Reidemeister moves; see $\S 7$.

A triangle in $M$ defines a trivial knot, and a loop in the plane with no crossings is said to be a trivial knot diagram. A knot diagram $\mathcal{D}$ is unknotted if it is equivalent to a trivial knot diagram under Reidemeister moves.

We measure the complexity of a knot diagram $\mathcal{D}$ by using its crossing number, the number of vertices in the planar graph $\mathcal{D}$; see $\S 7$. A problem of long standing is to determine an upper bound for the number of Reidemeister moves needed to transform an unknotted knot diagram $\mathcal{D}$ to the trivial knot diagram, as an explicit function of the crossing number $n$; see Welsh [30 p. 95]. This paper obtains such a bound.

Theorem 1.1. There is a positive constant $c_{1}$, such that for each $n \geq 1$, any unknotted knot diagram $\mathcal{D}$ with $n$ crossings can be transformed to the trivial knot diagram using at most $2^{c_{1} n}$ Reidemeister moves.

We obtain the explicit value of $10^{11}$ for $c_{1}$; this value can clearly be improved. The methods used in this paper do not lead to a small constant, so we chose a simplified exposition at the cost of obtaining a larger constant.

Received by the editors May 8, 1998 and, in revised form, October 10, 2000.

1991 Mathematics Subject Classification. Primary 57M25; Secondary 11Y16, 68W40.

Key words and phrases. Knot theory, knot diagram, Reidemeister move, normal surfaces, computational complexity.

The first author was partially supported by NSF grant DMS-9704286.

This paper grew out of work begun while the authors were visiting the Mathematical Sciences Research Institute in Berkeley in 1996/97. Research at MSRI was supported in part by NSF grant DMS-9022140. 


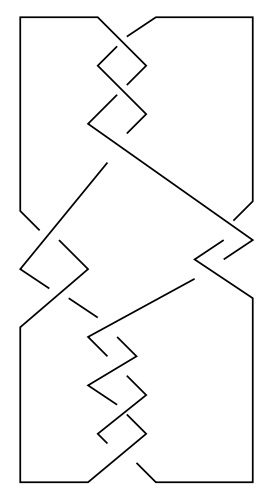

FiguRE 1. Goeritz's unknot.

In 1934 Goeritz [9] found diagrams $\mathcal{D}$ of the unknot such that any sequence of Reidemeister moves converting $\mathcal{D}$ to the trivial knot must pass through some intermediate knot diagram $\mathcal{D}^{\prime}$ that has more crossings than $\mathcal{D}$. (See Figure 1 )

It is not known how much larger the number of crossings needs to be for a general $n$-crossing knot, but Theorem 1.1 implies the following upper bound.

Corollary 1.1. Any unknotted knot diagram $\mathcal{D}$ with $n$ crossings can be transformed by Reidemeister moves to a trivial knot diagram through a sequence of knot diagrams each of which has at most $2^{c_{1} n}$ crossings.

This holds because each Reidemeister move creates or destroys at most two crossings, and all crossings must be eliminated by the final Reidemeister move. It seems plausible that for each knot diagram representing the unknot there exists some sequence of Reidemeister moves taking it to the trivial knot diagram which keeps the crossing number of all intermediate knot diagrams bounded by a polynomial in $n$, but this remains open.

In a general triangulated 3-manifold, a trivial knot is a triangle lying inside one tetrahedron and an elementary move takes place entirely within a single tetrahedron of the triangulation. The proof of Theorem 1.1 is based on a general result that bounds the number of elementary moves needed to transform a closed polygonal curve $K$ embedded in the 1-skeleton of a triangulated $P L$ 3-manifold $M$ to a trivial knot.

Theorem 1.2. There is a constant $c_{2}$ such that for each $t \geq 1$, any compact, orientable, PL 3-manifold with boundary $(M, \partial M)$ triangulated by tetrahedra has the following property: If $K$ is an unknot embedded in the 1-skeleton of $\operatorname{int}(M)$, then $K$ can be isotoped to the trivial knot using at most $2^{c_{2} t}$ elementary moves.

We obtain the explicit value $c_{2}=10^{7}$. The hypothesis that $K$ lies in the interior of $M$ can be weakened to require only that $K$ lies in $M$, as we indicate in $\S 6$.

The methods we use are based on the normal surface theory of Kneser [20] and Haken [10]. Haken applied this theory to obtain an algorithm to decide if a knot is trivial. See [11] for a survey of algorithms to recognize unknotting, and [5] and [4] for an alternate approach based on braids. A bound on the number of Reidemeister moves between a knot diagram and the unknot gives an algorithm to check unknottedness, based on checking the results of the finite number of possible configurations generated by at most this number of Reidemeister moves, and checking 
to see if any of them has no crossings. This is not a recommended approach, even if the bound on the number of Reidemeister moves were to be substantially improved. However it points to a close connection between the two questions.

The paper is organized as follows. In $\S 2$ we define elementary moves and outline the proof of Theorem 1.2 In $\S 3, \S 4$ and $\S 5$ we bound the number of elementary moves needed to isotop a curve in three distinct settings. First we consider an isotopy of a curve to a point across a compressing disk in a 3-manifold, secondly we consider an isotopy through a solid torus from a core to a longitude, and finally we consider an isotopy across a surface between two isotopic curves. In $\S 6$ we tie these results together to complete the proof of Theorem 1.2. We then consider the more special case of knots in $\mathbb{R}^{3}$. In $\S 7$ we outline the proof of Theorem 1.1 and begin it by relating elementary moves to Reidemeister moves. In $\S 8$ we start with a knot diagram and show how to construct a triangulation of a convex polyhedron of $\mathbb{R}^{3}$ containing the corresponding knot in its 1 -skeleton. This allows us to apply Theorem 1.2. In $\S 9$ we complete the proof of Theorem 1.1

After completing this paper we learned that S. Galatolo has obtained results for the case of knots in $\mathbb{R}^{3}$ of a similar nature to those stated in Theorem 1.1 and Corollary [1.1 and at about the same time. An announcement of his results appears in [8]. Galatolo's constructions are also based on an analysis of normal surfaces. The bound on the number of elementary moves announced in [8] is $O\left(2^{c n^{4}}\right)$.

\section{Elementary moves in a 3-MANifold}

In this section we define elementary moves for knots and links. Elementary moves are well defined in arbitrary triangulated $P L 3$-manifolds. In section 7 we will discuss Reidemeister moves, which are associated to knots and links in $\mathbb{R}^{3}$, and relate these two concepts for manifolds $P L$-embedded in $\mathbb{R}^{3}$.

A (piecewise-linear, unoriented) knot $K$ in a triangulated $P L$ manifold $M$ is a closed embedded polygonal curve. An (unoriented) link $L$ is a finite union of non-intersecting knots in $M$. Let $|L|$ denote the number of vertices of $L$.

An elementary move (or $\Delta$-move) on a knot or link consists of one of the following operations (see Burde and Zieschang [6, p. 4], Murasugi [21, p. 7]). (See Figure 2]) Each of these operations is required to take place in a single tetrahedron of $M$.

(1) Split an edge into two edges by adding a new vertex to its interior.

$\left(1^{\prime}\right)$ [Reverse of (1)] Combine two edges whose union is a line segment, erasing their common vertex.

(2) Given a line segment $A B$ of $L$ that contains a single interior vertex $D$ and a point $C$ not in $L$ such that the triangle $A B C$ intersects $L$ only in the edge $A B$, erase edge $A B$ and add two new edges $A C$ and $B C$ and a new vertex $C$.

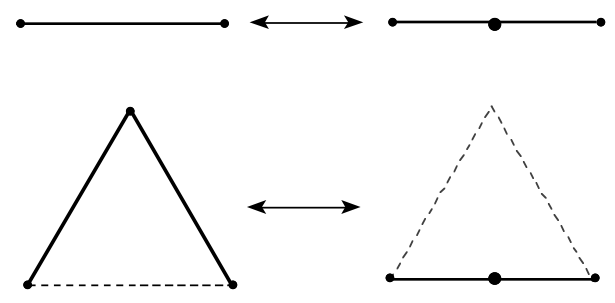

Figure 2. Type (1) and type (2) elementary moves. 
$\left(2^{\prime}\right)$ [Reverse of (2)] If for two adjacent edges $A C$ and $B C$ of $K$ the triangle $A B C$ intersects $L$ only in $A C$ and $B C$, then erase $A C, B C$ and vertex $C$ and add the edge $A B$, with a new interior vertex $D$.

Corresponding to elementary moves of type $(2),\left(2^{\prime}\right)$ is the piecewise-linear operation of linearly pulling the edges of a link across a triangle $A B C$. To define this operation, an extra vertex $D$ on the segment $A B$ must be specified; this is the role of elementary moves $(1)$ and $\left(1^{\prime}\right)$.

Two knots $K_{1}$ and $K_{2}$ are equivalent if one can be reached from the other by a finite sequence of elementary moves. This gives the same equivalence classes as requiring $K_{1}$ and $K_{2}$ to be ambient isotopic in $M$ [25]. The unknot is the class of knots equivalent to a triangle contained in a tetrahedron, and a knot $K$ is unknotted if it is equivalent to the unknot. Equivalently, an unknot is the boundary of a $P L$ embedded disk in $M$ 25. We will work with knots embedded in the interior of $M$. This is not a significant restriction, since it is possible to perturb any knot which meets $\partial M$ into the interior of $M$, after some simplicial subdivision.

We conclude this section by outlining the proof of Theorem 1.2 We first construct a compact triangulated $P L$ submanifold $M_{K}$ by removing a regular neighborhood $R_{K}$ of $K$. This gives a new triangulated manifold $M_{K}=M-R_{K}$ whose boundary consists of the original boundary $\partial M$ plus a 2-torus $\partial R_{K}$, called the peripheral torus.

If $K$ is unknotted, then $M_{K}$ contains an essential normal disk $S$. Essential means that $\partial S \subseteq \partial R_{K}$ is not the boundary of any disk in the torus $\partial R_{K}$. Drawing on Haken's normal surface theory, a result of Hass, Lagarias and Pippenger [12] implies that $S$ can be chosen to contain at most $2^{8 t+6}$ triangles. We present this argument for completeness in $\S 3$. Its boundary $\partial S$ is a curve $K_{2} . K_{2}$ is isotopic to $K$ within $\bar{R}_{K}$. There is a sequence of at most $2^{8 t+7}$ elementary moves which deform $K_{2}$ to a triangle across triangles in $S$. This is carried out in $\S 3$. It remains to relate $K_{2}$ to $K$ by elementary moves.

In $\S 3$ we construct a curve $K_{1}$ isotopic to $K$ in $R_{K}$ which is a longitude, a curve on the peripheral torus, $\partial R_{K}$, which bounds an embedded disk in $M_{K}$ but not inside the peripheral torus. Such curves exist if $K$ is unknotted in $M$. In $\S 4$ we construct a longitude $K_{2}$ that lies in the 1-skeleton of the peripheral 2-torus $\partial R_{K}$, consists of at most $O\left(2^{c_{3} t}\right)$ edges and can be isotoped to $K$ using at most $O\left(2^{c_{3} t}\right)$ elementary moves. In $\S 5$ we prove results which are used to show that there is a sequence of at most $O\left(2^{c_{4} t}\right)$ elementary moves which deform $K_{1}$ to $K_{2}$ while remaining entirely in the peripheral 2-torus $\partial R_{K}$. In $\S 6$ we combine these results to complete the proof of Theorem 1.2. All the constants and bounds above are explicitly computed.

The proof of Theorem 1.2 in effect deforms the original knot $K$ to a trivial knot with elementary moves made inside three surfaces: an annulus is used to go from $K$ to the longitude $K_{1}$, a 2-torus is used to go from $K_{1}$ to the boundary of a normal disk $K_{2}$, and a disk is used to go from $K_{2}$ to the trivial knot.

\section{NORMAL SURFACES AND ELEMENTARY MOVES ACROSS AN ESSENTIAL DISK}

In this section we assume given a compact $P L$ 3-manifold with (possibly empty) boundary $(M, \partial M)$, which is triangulated using $t$ tetrahedra. We will work with a knot $K$ embedded in the 1-skeleton of $\operatorname{int}(M)$. By barycentrically subdividing $M$ twice, we obtain a manifold $\left(M^{\prime \prime}, \partial M^{\prime \prime}\right)$ in which $K$ has a regular neighborhood $R_{K}$. The regular neighborhood, obtained by taking the closed star of $K$ in $M^{\prime \prime}$, 
is a solid torus with $\partial R_{K}$ disjoint from $\partial M$. We let $M_{K}=\left(M^{\prime \prime} \backslash R_{K}\right) \cup \partial R_{K}$ and note that its boundary $\partial M_{K}=\partial M^{\prime \prime} \cup \partial R_{K}$. We call the manifold $\left(M_{K}, \partial M_{K}\right)$, together with a marking of $\partial R_{K}$, a truncated knot complement for $K$. The marking serves to distinguish the peripheral torus from other boundary components of $M_{K}$, if any.

A meridian on the peripheral torus $\partial R_{K}$ is an oriented simple closed curve $\mu$ whose homology class $[\mu]$ in $H_{1}\left(\partial R_{K}, \mathbb{Z}\right)$ generates the kernel of the map

$$
j_{*}: H_{1}\left(\partial R_{K}, \mathbb{Z}\right) \rightarrow H_{1}\left(R_{K}, \mathbb{Z}\right),
$$

where $j: \partial R_{K} \hookrightarrow R_{K}$ is the inclusion map. Equivalently, it is a simple closed curve which bounds a disk in $R_{K}$ but not in $\partial R_{K}$.

A longitude on the peripheral torus $\partial R_{K}$ is an oriented simple closed curve $\lambda$ which intersects a meridian transversely at a single point and whose homology class $[\lambda]$ in $H_{1}\left(\partial R_{K}, \mathbb{Z}\right)$ generates the kernel of the map

$$
i_{*}: H_{1}\left(\partial R_{K}, \mathbb{Z}\right) \rightarrow H_{1}\left(M_{K}, \mathbb{Z}\right),
$$

where $i: \partial R_{K} \hookrightarrow M_{K}$ is the inclusion map, provided that this kernel is non-trivial. Note that the kernel is always infinite cyclic or trivial 6 .

If the core $K$ of the solid torus $R_{K}$ is unknotted in $M$, then a longitude exists, and it bounds a disk in $M_{K}$. For general $K$, a longitude does not exist when $K$ represents an element of infinite order in $H_{1}(M, \mathbb{Z})$. If $M \cong S^{3}$, or more generally a homology sphere, then a longitude exists for any $K$. This curve is sometimes called a preferred longitude.

When a longitude $\lambda$ of $\partial R_{K}$ exists, its intersection number on $\partial R_{K}$ with a meridian $\mu$ is \pm 1 , and the homology classes $[\mu]$ and $[\lambda]$ generate $H_{1}\left(\partial R_{K}, \mathbb{Z}\right) \cong$ $\mathbb{Z} \oplus \mathbb{Z}$.

A properly embedded disk in a 3-manifold with boundary $(M, \partial M)$ is a disk $S$, $P L$-embedded in $M$ with $S \cap \partial M=\partial S$. An essential disk in $M$ is a properly embedded disk such that $\partial S$ does not bound a disk in $\partial M$, i.e. the homotopy class of $\partial S$ in $\pi_{1}(\partial M)$ is non-trivial.

Lemma 3.1. Let $M_{K}$ be a triangulated PL 3-manifold which is a truncated knot complement for $K$. Then $K$ is unknotted if and only if $M_{K}$ contains an essential disk $S$ with $\partial S$ a longitude in $\partial R_{K}$.

Proof. If $K$ is unknotted, then it bounds a disk which is embedded in $M$. This disk can be intersected with $M_{K}$ to give an essential $P L$ disk $S$ in $M_{K}$. Conversely if $M_{K}$ contains an essential $P L$ disk $S$ with $\partial S \subseteq \partial R_{K}$, then the boundary $\partial S$ of $S$ represents a longitude on $\partial R_{K}$, which is isotopic to $K$ in $R_{K}$ 6. p. 29, Theorem 3.1], and therefore is equivalent to $K$. This disk can be used to contract $\partial S$ until it becomes a single triangle in $M_{K}$. The first homology group of a torus, $Z \oplus Z$, is canonically isomorphic to its fundamental group, and the statement about the homology class of $[\partial S]$ is a consequence.

We will use normal surface theory in the form described in Jaco and Rubinstein [18, Section 1]. A normal surface $S$ in a triangulated compact 3 -manifold $M$ is a $P L$-surface whose intersection with each tetrahedron in $M$ consists of a disjoint set of elementary disks. These are either triangles or quadrilaterals. A quadrilateral consists of two triangles glued together along an edge lying interior to the 


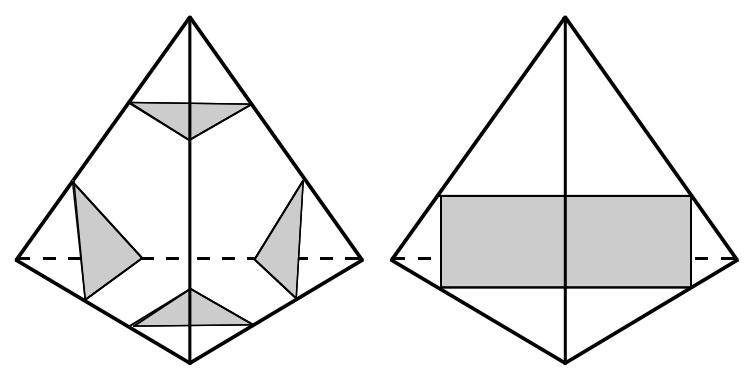

FIgURE 3. Elementary disks in a normal surface.

tetrahedron. All other edges of each triangle or quadrilateral are contained in the 2-skeleton of the tetrahedron, and do not intersect any vertices. (See Figure 3)

Within each tetrahedron of $M$ there are only seven kinds of possible triangles or $P L$ quadrilaterals, up to $P L$-isotopies that map the tetrahedron to itself. These elementary disks are labeled by the seven possible ways of partitioning the vertices of the tetrahedron into the two non-empty sets determined by cutting the tetrahedron along the triangle or quadrilateral. W. Haken observed that a normal surface is determined (up to isotopy, and in fact up to an even stricter equivalence called normal isotopy) by the number of pieces of each of the seven kinds of elementary disks that occur in each tetrahedron* This can be described by a non-negative integer vector $\mathbf{v}=\mathbf{v}(S) \in \mathbb{Z}^{7 t}$, which gives the normal coordinates of $S$. He also showed that the set of allowable values $\mathbf{v}(S)$ for a normal surface $S$ lies in a certain homogeneous rational cone $\mathcal{C}_{M}$ in $\mathbb{R}^{7 t}$ which we call the Haken normal cone. If $\mathbf{v}=\left(v_{1}, v_{2}, \ldots, v_{7 t}\right) \in \mathbb{R}^{7 t}$, then Haken's normal cone is specified by a set of linear equations and inequalities of the form

$$
\begin{gathered}
v_{i} \geq 0, \quad \text { for } 1 \leq i \leq 7 t \\
v_{i_{1}}+v_{i_{2}}=v_{i_{3}}+v_{i_{4}} \quad \text { (up to } 6 t \text { equations). }
\end{gathered}
$$

The second set of equations expresses matching conditions which say that the number of edges on a common triangular face of two adjacent tetrahedra coming from regions in each of the tetrahedra must match. For each triangular face there are three types of edges (specified by a pair of edges on the triangle), which yield three matching conditions per face. Triangular faces in the boundary $\partial M$ give no matching equations. The cone $\mathcal{C}_{M}$ is rational because all equations (3.1), (3.2) have integer coefficients. We let

$$
\mathcal{C}_{M}(\mathbb{Z})=\mathcal{C}_{M} \cap \mathbb{Z}^{7 t}
$$

which is the set of integral vectors in the Haken normal cone $\mathcal{C}_{M}$. Haken defined a fundamental surface to be a normal surface $S$ such that

$$
\mathbf{v}(S) \neq \mathbf{v}_{1}+\mathbf{v}_{2}, \quad \text { with } \mathbf{v}_{1}, \mathbf{v}_{2} \in \mathcal{C}_{M}(\mathbb{Z}) \backslash\{\mathbf{0}\} .
$$

Such a vector $\mathbf{v}(S)$ is called an element of the minimal Hilbert basis $\mathcal{H}\left(\mathcal{C}_{M}\right)$ of $\mathcal{C}_{M}$, using the terminology of integer programming; see Schrijver [27, Theorem 16.4].

*Haken's [10] treatment of normal surfaces was based on a formulation using cells. We follow an alternate development based on tetrahedra, as in Jaco and Rubinstein [18, Section 1] and Jaco and Tollefson [19]. 
Theorem 3.1 (Haken). If a triangulated, irreducible, orientable, compact 3-manifold with boundary $(M, \partial M)$ contains an essential disk whose boundary is in a component $\partial R$ of $\partial M$, then it contains such a disk $S$ which is a fundamental normal surface.

Proof. See Jaco-Rubinstein [18, Theorem 2.3] for a treatment of the existence of an essential normal disk in $M$ with boundary on $\partial R$. For the existence of a fundamental disk, we refer to Jaco and Tollefson [19, Corollary 6.4], which establishes the stronger result that there exists such a disk that is a fundamental surface and is also a vertex surface, which means that $\mathbf{v}(S)$ lies on an extreme ray of Haken's normal cone $\mathcal{C}_{M}$.

Remark. This result applies in arbitrary 3-manifolds. The proof in [19] assumes compactness and irreducibility of $M$, but these hypotheses can be removed. A classical theorem of Alexander implies that knot complements in the 3-sphere are always irreducible.

Hass, Lagarias and Pippenger [12] give a simple bound for the complexity of any fundamental surface, whose proof we include for completeness.

Lemma 3.2. Let $M$ be a triangulated compact 3-manifold, possibly with boundary, that contains t tetrahedra.

(1) Any vertex minimal solution $\mathbf{v} \in \mathbb{Z}^{7 t}$ of the Haken normal cone $\mathcal{C}_{M}$ in $\mathbb{R}^{7 t}$ has

$$
\max _{1 \leq i \leq 7 t}\left(v_{i}\right) \leq 2^{7 t-1}
$$

(2) Any minimal Hilbert basis element $\mathbf{v} \in \mathbb{Z}^{7 t}$ of the Haken fundamental cone $\mathcal{C}_{M}$ has

$$
\max _{1 \leq i \leq 7 t}\left(v_{i}\right) \leq t \cdot 2^{7 t+2}
$$

Proof. (1) Choose a maximal linearly independent subset of the matching condition equations (3.2). There will be $7 t-d$ of them, where $d=\operatorname{dim}_{\mathbb{R}}\left(\mathcal{C}_{M}\right)$. Any vertex ray is determined by adjoining to these equations $d-1$ other binding inequality constraints

$$
\left\{v_{i_{k}}=0 ; 1 \leq k \leq d-1\right\}
$$

with the proviso that the resulting system have rank $7 t-1$. These conditions yield a $(7 t-1) \times 7 t$ integer matrix $\mathrm{M}$ of rank $7 t-1$, and the vertex ray elements $\mathbf{z}=\left(z_{1}, \ldots, z_{7 t}\right)$ corresponding to $\mathrm{M}$ are the real solutions to

$$
\mathrm{Mz}=\mathbf{0} .
$$

In order to get a feasible vertex ray in $\mathcal{C}_{M}$, all non-zero coordinates $z_{i}$ of $\mathbf{z}$ must have positive sign.

At least one of the unit coordinate vectors $\mathbf{e}_{1}, \mathbf{e}_{2}, \ldots, \mathbf{e}_{7 t}$ must be linearly independent of the row space of M. Adjoin it as a first row to $\mathrm{M}$ and we obtain a full rank $7 t \times 7 t$ integer matrix

$$
\tilde{M}=\left[\begin{array}{c}
\mathbf{e}_{k} \\
\mathrm{M}
\end{array}\right], \quad \operatorname{det}(\tilde{M}) \neq 0 .
$$


Consider the adjoint matrix $\operatorname{adj}(\tilde{\mathrm{M}})=\operatorname{det}(\tilde{\mathrm{M}}) \tilde{\mathrm{M}}^{-1}$, which has integer entries

$$
w_{i j}=\operatorname{adj}(\tilde{\mathrm{M}})_{i j}=(-1)^{i+j} \operatorname{det} \tilde{\mathrm{M}}[j \mid i],
$$

in which $\tilde{\mathrm{M}}[j \mid i]$ is the minor obtained by crossing out the $j^{\text {th }}$ row and $i^{\text {th }}$ column of $\tilde{M}$. Let

$$
\mathbf{w}=\left[w_{11}, w_{21}, \ldots, w_{7 t, 1}\right]^{t}
$$

be the first column of $\operatorname{adj}(\tilde{\mathrm{M}})$. Since $\tilde{\mathrm{M}} a \operatorname{dj}(\tilde{\mathrm{M}})=\operatorname{det}(\tilde{\mathrm{M}}) \mathrm{I}$, this yields

$$
\mathrm{Mw}=\mathbf{0},
$$

and $\mathbf{w} \neq \mathbf{0}$ because $\left\langle\mathbf{e}_{k}, \mathbf{w}\right\rangle=\operatorname{det}(\tilde{\mathrm{M}}) \neq 0$. We bound the entries of $\mathbf{w}$ using Hadamard's inequality, which states for an $m \times m$ real matrix $\mathrm{N}$ that

$$
\operatorname{det}(\mathrm{N})^{2} \leq \prod_{i=1}^{m}\left\|\mathbf{n}_{i}\right\|^{2},
$$

in which $\left\|\mathbf{n}_{i}\right\|^{2}$ is the Euclidean length of the $i^{\text {th }}$ row $\mathbf{n}_{i}$ of N. We apply this to equation (3.6), and observe that each row of the $(7 t-1) \times(7 t-1)$ matrix $\tilde{\mathrm{M}}[j \mid i]$ has squared Euclidean length at most 4, because this is true for all row vectors in the system of equations (3.2) and for $\mathbf{e}_{k}$. Applied to equation (3.6) this gives

$$
\left|w_{i j}\right|^{2} \leq 4^{7 t-1} \text {. }
$$

Now equation (3.6) shows that $\mathbf{w} \in \mathbb{Z}^{7 t}$, and a vertex minimal solution $\mathbf{v}$ in the extreme ray is obtained by dividing $\mathbf{w}$ by the greatest common divisor of its elements, hence

$$
\max _{1 \leq i \leq 7 t}\left|v_{i}\right| \leq \max _{1 \leq i \leq 7 t}\left|w_{i 1}\right| \leq 2^{7 t-1} .
$$

(2) A simplicial cone $\mathcal{C}$ in $\mathbb{R}^{7 t}$ is a $d$-dimensional pointed cone which has exactly $d$ extreme rays. Let $\mathbf{v}^{(1)}, \ldots, \mathbf{v}^{(d)} \in \mathbb{Z}^{7 t}$ be the vertex minimal solutions for the extreme rays. Each point in $\mathcal{C}$ can be expressed as a non-negative linear combination of the $\mathbf{v}^{(j)}$, as

$$
\mathbf{v}=\sum_{j=1}^{d} c_{j} \mathbf{v}^{(j)}, \quad \text { each } \quad c_{j} \geq 0 .
$$

If $\mathbf{v}$ is in the minimal Hilbert basis $\mathcal{H}(\mathcal{C})$ of the cone $\mathcal{C}$, then $0 \leq c_{j} \leq 1$, for otherwise one has

$$
\mathbf{v}=\left(\mathbf{v}-\mathbf{v}^{(j)}\right)+\mathbf{v}^{(j)},
$$

and both $\mathbf{v}-\mathbf{v}^{(j)}$ and $\mathbf{v}^{(j)}$ are non-zero integer vectors in $\mathcal{C}$, which is a contradiction. Thus, any minimal Hilbert basis element $\mathbf{v}=\left(v_{1}, \ldots, v_{7 t}\right)$ of a simplicial cone satisfies

$$
\left|v_{i}\right| \leq \sum_{j=1}^{d}\left|v_{i}^{(j)}\right| \quad \text { for } \quad 1 \leq i \leq 7 t .
$$

The Haken normal cone $\mathcal{C}_{M}$ may not be simplicial, but we can partition it into a set of simplicial cones $\left\{\mathcal{C}_{m}^{(k)}\right\}$ each of whose extreme rays are extreme rays of $\mathcal{C}_{M}$ itself. We have

$$
\mathcal{H}\left(\mathcal{C}_{M}\right) \subseteq \bigcup_{k} \mathcal{H}\left(\mathcal{C}_{M}^{(k)}\right)
$$


Thus all Hilbert basis elements of $\mathcal{H}\left(\mathcal{C}_{M}\right)$ satisfy the bound (3.8) for Hilbert basis elements of $\mathcal{H}\left(\mathcal{C}_{M}^{(k)}\right)$. Using equation (3.4) to bound $\left|v_{i}^{(j)}\right|$ we obtain

$$
\left|v_{i}\right| \leq d 2^{7 t-1} \leq 7 t 2^{7 t-1} \leq t 2^{7 t+2}
$$

as claimed.

A $P L$-embedded normal disk $S$ can be used as a template to transform its boundary $\partial S$ to a single triangle by a sequence of elementary moves, in such a way that all intermediate curves lie on the surface $S$.

Lemma 3.3. Let $M$ be a piecewise-linear triangulated compact 3-manifold with boundary, and let $S$ be a normal disk in $M$ that consists of $w$ triangles. Then $\partial S$ can be isotoped to a triangle by a sequence of at most $2 w$ elementary moves on $M$, each of which takes place in a triangle or edge contained within $S$.

Proof. We will construct a sequence of $w$ simple closed curves

$$
\partial S=C_{0}, C_{1}, C_{2}, \ldots, C_{w-1}=\partial T
$$

where $T$ is a triangle in $S$, and such that for $0 \leq i \leq w-1$ :

(i) $C_{i+1}$ is obtained from $C_{i}$ by at most two elementary moves; either a move of type $\left(2^{\prime}\right)$ across a single triangle in $S$ followed by a move of type $\left(1^{\prime}\right)$ which removes the extra vertex created by the first move, or a move of type (1) followed by a move of type (2) across a single triangle in $S$.

(ii) There is a triangulated disk $S_{i}$ contained in $S$ which consists of $w-i$ triangles, such that $C_{i}=\partial S_{i}$.

The construction proceeds by eliminating triangles in $S$ one at a time by a sequence of steps, each consisting of two elementary moves, in such a way as to preserve the property that each $S_{j}$ is a topological disk. We proceed by induction on $j$, with the base case $j=0$ holding by hypothesis. For the induction step, we note that the only way that removing from $S_{j}$ a triangle that contains at least one edge of $\partial S_{j}$ can yield a surface $S_{j+1}$ that is not a topological disk is by producing a vertex $\mathbf{v}$ that is a cut-point, i.e. $\mathbf{v}$ is visited twice in the curve $\partial S_{j+1}$. In that case $\mathbf{v}$ is a vertex of $\partial S_{j}$, which implies that the triangle that is removed must have exactly one edge on $\partial S_{j}$, and the pair of elementary moves pulls this edge across to the two edges of this triangle that are adjacent to $\mathbf{v}$. We therefore proceed by first checking if $S_{j}$ has any triangle having two edges on $\partial S_{j}$. If so, we remove this triangle to obtain $S_{j+1}$. If this cannot be done, then $S_{j}$ must contain an interior vertex, because a triangulated polygon that has no interior vertices and contains more than one triangle contains at least 2 triangles having two edges on the boundary. In this case there exists a triangle having one edge on the boundary which also has an interior vertex, and we can pull across this triangle to obtain $S_{j+1}$. This completes the induction step.

Putting all these results together yields:

Lemma 3.4. Let $M_{K}$ be a triangulated PL 3-manifold consisting of t tetrahedra which is a truncated knot complement for $K$. If $K$ is unknotted, then there is an essential normal disk $S$ in $M_{K}$ that contains at most $2^{8 t+6}$ triangles, and $S$ has a polygonal boundary $K_{1}=\partial S$ which lies on the peripheral 2-torus $\partial R_{K}$ and consists of at most $2^{8 t+7}$ edges. The boundary $K_{1}$ of $S$ can be transformed to a single 
triangle by at most $2^{8 t+7}$ elementary moves, with all intermediate curves being the boundary of some triangulated subdisk of $S$.

Proof. By Lemma 3.1 and Theorem 3.1 there exists an essential normal disk which is a fundamental surface for $M_{K}$. The bound of Lemma 3.2 implies that this surface contains at most $7 t^{2} \cdot 2^{7 t+2}<2^{8 t+5}$ triangles and quadrilaterals. Since each quadrilateral consists of two triangles in the triangulation, we obtain the upper bound $2^{8 t+6}$ for the total number of triangles. The boundary $\partial S$ contains at most two edges of each triangle, for a total of at most $2^{8 t+7}$ edges. Lemma 3.3 gives the stated bound on elementary moves.

\section{LONGITUDES AND CORES OF SOLID TORI}

In this section we bound the number of elementary moves needed to isotop a knot $K$ in the 1-skeleton of a triangulated compact 3-manifold $(M, \partial M)$ across an annulus to a curve $K_{2}$ which is a longitude on the peripheral torus $\partial R_{K}$ of $K$. The main result of this section is the following:

Theorem 4.1. Let $(M, \partial M)$ be a triangulated compact PL 3-manifold with t tetrahedra, let $K$ be an unknotted simple curve in the 1-skeleton of $\operatorname{int}(M)$, and let $\partial R_{K}$ be the peripheral torus of $K$ in the second barycentric subdivision of $M$. Then there exists a PL closed curve $K_{2}$ in $\partial R_{K}$ which is a longitude for $\partial R_{K}$ in $M_{K}$ such that:

(i) There is an embedded $P L$ annulus $S$ in the solid torus $R_{K}$ whose two boundary components are $K_{2}$ and $K$, and which consists of at most $2^{1858 t}$ triangles.

(ii) There is an isotopy from $K_{2}$ to $K$ across $S$ that consists of at most $2^{1858 t+1}$ elementary moves.

Remarks. The closed curve $K_{2}$ is embedded in $\partial R_{K}$ but generally does not lie in its 1-skeleton. In the special case that $(M, \partial M)$ is $P L$-homeomorphic to a triangulated convex polyhedron in $\mathbb{R}^{3}$, as occurs with a standard knot, the exponential bounds in (i), (ii) can be improved to bounds polynomial in $t$, as we indicate after the proof.

The proof of Theorem 4.1 proceeds in three steps. We first construct a closed curve $\alpha$ embedded in the 1-skeleton of $R_{K}$ which is parallel to the core $K$, i.e. its homology class $[\alpha]$ in $H_{1}\left(\partial R_{K}, \mathbb{Z}\right)$ has intersection number \pm 1 with a meridian $[\mu]$. We next observe that the homology class in $H_{1}\left(\partial R_{K}, \mathbb{Z}\right)$ of a longitude $K_{2}$ is necessarily

$$
\left[K_{2}\right]= \pm([\alpha]+k[\mu])
$$

for some integer $k$. The second step is to bound $|k|$ by an exponential function of $t$. The third step is to obtain $K_{2}$ from $\alpha$ by adding $k$ twists to $\alpha$ near a fixed meridian $\mu$. Given $K_{2}$, we construct a triangulated annular surface $S$ embedded in $R_{K}$ which lies in the 2-skeleton of $R_{K}$ except in a "collar" of one meridian disk, into which the $k$ twists are inserted. All vertices of the triangulated annulus $S$ lie in its boundary $\partial S=K_{2} \cup K$. Finally we obtain an isotopy giving the elementary move bound by pulling $K$ across the triangles in $S$ to $K_{2}$.

We begin with some preliminary lemmas involving $P L$ topology, which pin down some of the combinatorial structure of the regular neighborhood of $K$. Background for $P L$ topology can be found in [17] or [26]. 
Let $v_{0}, \ldots, v_{r}$ be the vertices of the knot $K$ in $M$. In the process of taking the second barycentric subdivision of $M$, each edge $\left[v_{j}, v_{j+1}\right]$ of $K$ is subdivided into four edges by the addition of three new vertices. We continue to denote the subdivided curve by $K$. Denote the original vertices $v_{i}$ by $w_{4 i}$ and let $w_{4 i+1}, w_{4 i+2}, w_{4 i+3}$, $0 \leq i \leq r$, denote the new vertices added in the two barycentric subdivisions. We also use the convention that $w_{j+s}=w_{j}$, where $s=4 r+4$ is the total number of vertices, so that the vertices $w_{i}$ of $K$ are cyclically ordered.

We break up the solid torus $R_{K}$ into a union of what we call wheels and wedges. For each edge $\left[w_{i-1}, w_{i}\right]$ let the wheel around that edge be the closed star $S t\left(\left[w_{i-1}, w_{i}\right], R_{K}\right)$ of the edge $\left[w_{i-1}, w_{i}\right]$ in the solid torus $R_{K}$. This is the union of all the tetrahedra in $R_{K}$ having $\left[w_{i-1}, w_{i}\right]$ as an edge, and is homeomorphic to a closed ball. For each vertex $w_{i}$ on $K$ let the wedge around $w_{i}$ be the union of all the tetrahedra in $R_{K}$ meeting $K$ only at the point $w_{i}$. Let

$$
\mu_{i}=l k\left(\left[w_{i-1}, w_{i}\right], R_{K}\right), \quad 1 \leq k \leq s,
$$

be the link of the edge $\left[w_{i-1}, w_{i}\right]$ in the solid torus $R_{K}$.

Lemma 4.1. (1) Each $\mu_{i}$ lies in $\partial R_{K}$ and is a meridian.

(2) $\mu_{i}$ and $\mu_{j}$ coincide if $i$ is odd and $j=i+i$, or if $i$ is even and $j=i-1$. Otherwise $\mu_{i}$ and $\mu_{j}$ are disjoint.

(3) An edge on $\partial R_{K}$ either lies on some $\mu_{i}$ or else lies on a unique triangle in $R_{K}$ whose opposite vertex $w$ lies on $K$.

Proof. (1) The closed star $S t\left(\left[w_{i-1}, w_{i}\right], R_{K}\right)$ is supported on the set of tetrahedra in $R_{K}$ having $\left[w_{i-1}, w_{i}\right]$ as an edge. Each such tetrahedron has one edge which is disjoint from $\left[w_{i-1}, w_{i}\right]$, and the link $\mu_{i}$ is the union of these edges. The surface $D_{i}$ which is obtained by coning $\mu_{i}$ to the vertex $w_{i}$ forms a topological disk in $R_{K}$ whose boundary is $\mu_{i}$, and which intersects $K$ transversely in a single point. Thus $\mu_{i}$ is a meridian; see Figure 5

(2) See Figure 4 for a cross-sectional sketch of $R_{K}$. The vertices $w_{i}$ with $i$ odd were added during the second barycentric subdivision. Prior to the second subdivision, these vertices lay along interior points of edges of $K$. The two meridians associated to the edges $\left[w_{i-1}, w_{i}\right]$ and $\left[w_{i}, w_{i+1}\right]$ coincide for $i$ odd. We will show they are disjoint for $i$ even. For $j=0(\bmod 4)$ the distance (minimal number of edges in the 1-skeleton) between $w_{j}$ and $w_{j+4}$ in $R_{K}$ is four, since barycentric subdivision doubles distances between vertices. If $\mu_{j+2} \cap \mu_{j+3} \neq \emptyset$, then this distance would be two, since $\mu_{j+2}$ has distance one from $w_{j}$ and $\mu_{j+4}$ has distance one from $w_{j+4}$. Similarly the distance between $w_{j-1}$ and $w_{j+1}$ in $\operatorname{lk}\left(w_{j}\right)$ is at least

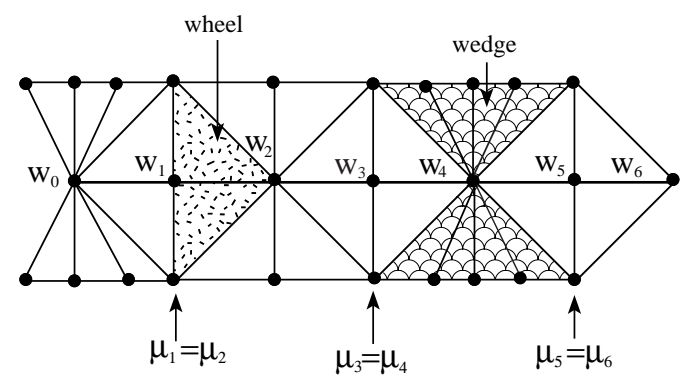

FiguRE 4. A 2-dimensional section of $R_{K}$. 


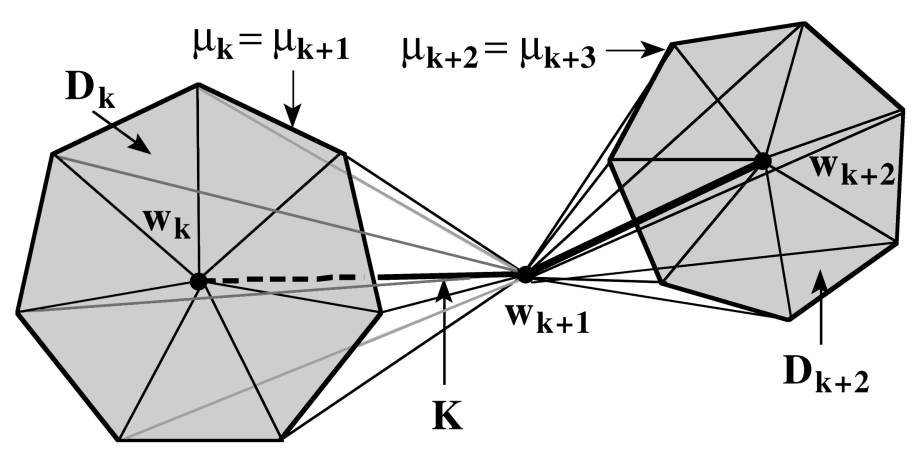

Figure 5. Adjacent meridians. The index $k$ is odd.

four, but it would be at most two if $\mu_{j} \cap \mu_{j+1} \neq \emptyset$. The intersection properties of the meridians follow.

(3) Each edge in $\partial R_{K}$ is an edge of a triangle with a vertex $w_{i}$ on $K$, since $R_{K}$ is obtained by taking the closed star of $K$ in $M^{\prime \prime}$. If the edge is in a meridian $\mu_{i}$, then it lies in triangles with vertices at both $w_{i-1}$ and $w_{i}$ or $w_{i}$ and $w_{i+1}$. If it doesn't lie on a meridian, then it is contained in the wedge around $w_{i}$. Any triangle containing the edge and a point $w^{\prime}$ on $K$ cannot cross the two wheels meeting $w_{i}$, and the point $w^{\prime}$ must coincide with $w$.

Lemma 4.2. There exists an embedded closed curve $\alpha$ in the 1-skeleton of $\partial R_{K}$ which is parallel to $K$ in $R_{K}$. There is an annulus $S_{0}$ embedded in the 2-skeleton of the solid torus $R_{K}$ whose boundary components are $\alpha$ and $K$, and all vertices of $S_{0}$ lie in its boundary $\alpha \cup K$.

Proof. We construct $\alpha$ as the union of a sequence of arcs. For each odd $i, 1 \leq i \leq s$, we pick a path $\alpha_{i}$ in the 1 -skeleton of $\partial R_{K}$ that connects a vertex $x_{i}$ of $\mu_{i}=\mu_{i+1}$ to a vertex $x_{i+1}$ on $\mu_{i+2}=\mu_{i+3}$, does not cross $\mu_{i-1}$, and is shortest among all such paths. (We don't allow $\alpha_{i}$ to cross $\mu_{i-1}$ to prevent it from running between the curves in the wrong direction.) We identify $x_{s}$ and $x_{0}$ as before. Note that $\alpha_{i}$ is embedded and that it meets the collection of meridians only at its endpoints, since otherwise it can be shortened.

We then add for each odd $i$ a connecting path $\beta_{i}$ on $\mu_{i}$ from the endpoint $x_{i-1}$ of $\alpha_{i-2}$ to the initial point $x_{i}$ of $\alpha_{i}$, and we set

$$
\alpha=\beta_{1} \alpha_{1} \beta_{3} \alpha_{3} \beta_{5} \ldots \beta_{s-1} \alpha_{s-1}
$$

The path $\alpha$ is a closed curve, and is embedded in $\partial R_{K}$.

We construct the surface $S_{0}$ as follows. Each arc $\beta_{i}, 1 \leq i \leq s, i$ odd, lies on a meridian $\mu_{i}=\mu_{i+1}$ which is the boundary of a meridian disk $D_{i+1}$ centered at the vertex $w_{i+1}$ on $K$, and we cone $\beta_{i}$ to $w_{i+1}$ along $D_{i+1}$, obtaining a subdisk of $D_{i+1}$. For each edge $\alpha_{i}$ that lies on the boundary of the wedge around the vertex $w_{i+1}$, there is a unique triangle containing that edge and $w_{i+1}$. For fixed $i$, the union of these triangles forms a disk in $R_{K}$ meeting $K$ at the point $w_{i+1}$, and $\partial R_{K}$ along $\beta_{i} \cup \alpha_{i}$, and whose boundary consists of $\beta_{i} \cup \alpha_{i}$ together with two interior edges of $R_{K}$, one running from the initial point $x_{i-1}$ of $\beta_{i}$ to $w_{i+1}$ and one running from the endpoint $x_{i+1}$ of $\alpha_{i}$ to $w_{i+1}$. 


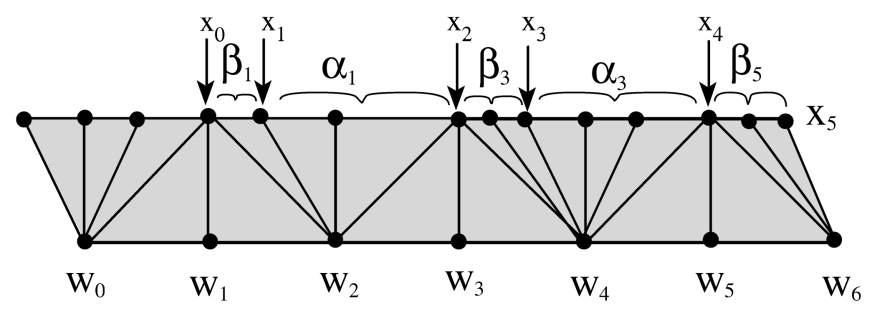

Figure 6 . The annulus $S_{0}$.

Finally for each meridian $\mu_{i}$ with $i$ odd, there is a pair of triangles

$$
\left[w_{i-1}, w_{i}, x_{i-1}\right]
$$

and

$$
\left[w_{i}, w_{i+1}, x_{i-1}\right]
$$

whose union is a disk contained in the two wheels around $\left[w_{i-1}, w_{i}\right]$ and $\left[w_{i}, w_{i+1}\right]$, and connecting the free edge running from the endpoint $x_{i-1}$ of $\alpha_{i-2}$ to $w_{i-1}$ to the free edge running from the initial point (also $x_{i-1}$ ) of $\beta_{i}$ to $w_{i+1}$. The union of all these disks forms an annulus which is embedded in the 2-skeleton of $R_{K}$, whose boundary components are $\alpha$ and $K$, and all vertices of which lie in $\alpha \cup K$. See Figure 6 .

Proof of Theorem 4.1. We wish to convert the closed curve $\alpha$ given in Lemma 4.2 to a longitude $K_{2}$ by adding "twists". The homology class $\left[K_{2}\right]$ of a longitude $K_{2}$ satisfies

$$
\left[K_{2}\right]= \pm([\alpha]+k[\mu])
$$

where $\mu$ is a fixed meridian. According to Lemma 4.1 we may choose $\mu$ to be $\mu_{1}$ taken with a fixed orientation.

The number $k$ represents the number of "twists" that we must insert in $\alpha$ to convert it to the knot $K_{2}$. We proceed to bound $k$ in terms of the number of tetrahedra $t$ in the original manifold $M$. We may assume $k \neq 0$ for otherwise we are done. The value of $k$ is uniquely determined by the requirement that the homology class $[\alpha]+k[\mu]$ vanish in $H_{1}\left(M_{K}, \mathbb{Z}\right)$. (Here we use the fact that $[\mu]$ is non-vanishing in $H_{1}\left(M_{K}, \mathbb{Z}\right)$.) This is equivalent to the curve $\alpha+k \mu$ being a boundary in the cycle group. The manifold $M_{K}$ contains at most $576 t$ tetrahedra, since it is contained in the second barycentric subdivision, hence the number $e$ of edges in $M_{K}$ is at most $3456 t$. The group of boundaries in the $P L$ chain complex of $M_{K}$ is generated by the paths around all 2-simplices in $M_{K}$, so the number $f$ of a linearly independent generating set of these is at most 2304t. We choose fixed orientations for the edges and faces, and we can then describe a basis of generators for the group of boundaries using an $e \times f$ matrix $\mathrm{M}=\left[\mathrm{M}_{a b}\right]$ with entries

$$
\mathrm{M}_{a b}= \begin{cases} \pm 1 & \text { if } a \text { is an edge in the oriented 2-simplex } b \\ 0 & \text { otherwise }\end{cases}
$$

in which each column of M has exactly three non-zero entries. Let $\mathbf{c}_{\alpha}$ be the $e \times 1$ vector describing $\alpha$ as an oriented cycle in $M_{K}$, and let $\mathbf{c}_{\mu}$ be the $e \times 1$ vector 
describing $\mu$ as an oriented cycle in $M_{K}$. All entries in $\mathbf{c}_{\alpha}$ and $\mathbf{c}_{\mu}$ are 0,1 or -1 . The condition for $\mathbf{c}_{\alpha}+k \mathbf{c}_{\mu}$ to be a boundary is that the linear system

$$
\mathrm{Mx}=\mathbf{c}_{\alpha}+k \mathbf{c}_{\mu}
$$

has a solution in integer unknowns $\mathbf{x}=\left(x_{1}, \ldots, x_{f}\right)^{T}$. The hypotheses imply that this linear system is solvable over $\mathbb{Z}$ for a unique value of $k$. Since we took a generating set of boundary faces, we have that $M$ is of full column rank. The meridian class $\mathbf{c}_{\mu}$ is not in the column space of $\mathrm{M}$ since otherwise $\mu$ would bound a surface in $M_{K}$ and the unknotted curve $K$ would represent an element of infinite order in $H_{1}(M, \mathbb{Z})$. It follows that there is at most one real value of $k$ for which the system (4.12) is solvable, for if there were two then $\mathbf{c}_{\mu}$ would be in the column space of M. Thus $\mathbf{c}_{\mu}$ is linearly independent of the column space of $\mathrm{M}$ and the matrix $\left[\mathrm{M}, \mathbf{c}_{\mu}\right]$ in the linear system

$$
\left[\mathrm{M}, \mathbf{c}_{\mu}\right]\left[\frac{\mathbf{x}}{-k}\right]=\mathbf{c}_{\alpha}
$$

has full column rank $f+1$. Choosing a suitable $f+1$ rows of $\left[\mathrm{M}, \mathbf{c}_{\mu}\right]$ yields an invertible matrix $\tilde{M}$ and

$$
\left[\frac{\mathbf{x}}{-k}\right]=\tilde{\mathrm{M}}^{-1} \tilde{\mathbf{c}}_{\alpha}
$$

in which $\tilde{\mathbf{c}}_{\alpha}$ is the subset of $\mathbf{c}_{\alpha}$ determined by the same $f+1$ rows. By Cramer's rule we have

$$
-k=\frac{1}{\operatorname{det}(\tilde{\mathrm{M}})} \sum_{i=1}^{f+1} \tilde{\mathrm{M}}[i, f+1]\left(\tilde{\mathbf{c}}_{\alpha}\right)_{i},
$$

where $\tilde{M}[i, f+1]$ denotes a minor of $\tilde{M}$ on the last column. Since $\left|\left(\tilde{\mathbf{c}}_{\alpha}\right)_{i}\right| \leq 1$ we have

$$
|k| \leq \sum_{i=1}^{f+1}|\tilde{\mathrm{M}}[i, f+1]| .
$$

We bound the terms in this expression using Hadamard's determinant bound (3.7). Each column of $\tilde{M}$ has at most three non-zero entries, each equal to \pm 1 , except the last column, which does not appear in the $f \times f$ minor $\tilde{\mathrm{M}}[i, f+1]$. This yields the bound

$$
|\tilde{\mathrm{M}}[i, f+1]|^{2} \leq 3^{f}, 1 \leq i \leq f+1,
$$

which with (4.13) yields

$$
|k| \leq(f+1) 3^{\frac{f}{2}} \leq(2304 t+1) 3^{1152 t} .
$$

We now describe how the "twists" are added to $\alpha$ and the surface $S_{0}$ of Lemma 4.2 to yield an annulus $S$ with boundary a longitude. The disk $D_{2}$ cuts the core $K$ at the point $w_{2}$ and stretches to the boundary. We add the twists to $S_{0}$ by modifying it inside the wedge around $w_{2}$. Let $A$ denote the surface consisting of the set of closed triangles in the wedge around $w_{2}$ which intersect the meridian $\mu_{2}$ and which lie in $\partial R_{K}$. Then $A$ is an annulus, and we suppose it contains $\ell$ triangles. The first edge $\left[x_{1}, v\right]$ of $\alpha_{1}$ has one endpoint on $\mu_{1}=\mu_{2}$ and the other endpoint disjoint from $\mu_{2}$ and on the other boundary component of $A$. We replace this edge with a path $\alpha^{\prime}$ of $k \ell$ edges which form a spiral going $k$ times around $A$, starting at $x_{1}$ and ending at $v$; see Figures 7 and $\left[8\right.$. To convert the surface $S_{0}$ of Lemma 4.2 to 


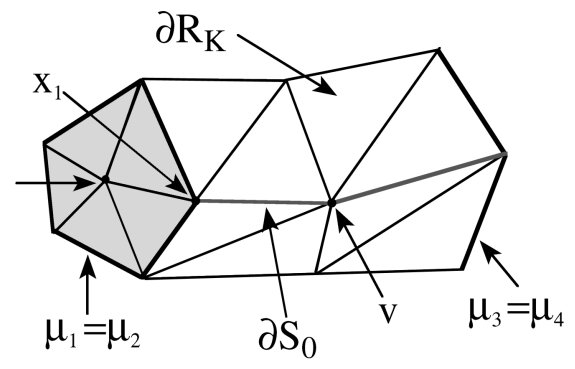

FiguRE $7 . S_{0}$.

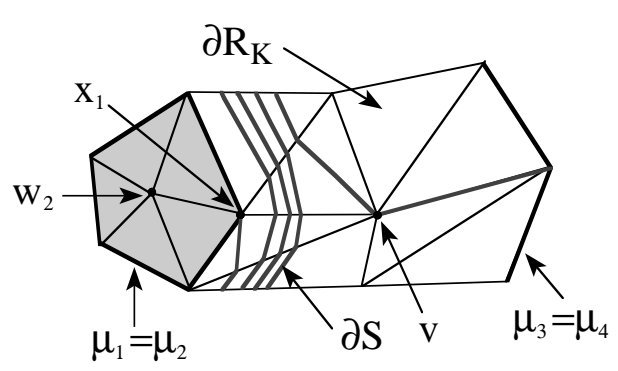

Figure 8. Adding twists replaces $S_{0}$ by $S$.

the desired surface $S$, we first subdivide the interval $\left[x_{1}, v\right]$ into $k \ell$ edges by adding vertices $y_{1}, y_{2}, \ldots, y_{k \ell-1}$. We remove the triangle $\left[x_{1}, v, w_{2}\right]$ from $S_{0}$ and add $k \ell$ new triangles, obtained by coning the edges of $\alpha^{\prime}$ to $w_{2}$. This construction produces the surface $S$, which is also an annulus and which also has its vertices either on $K$ or $\partial R_{K}$.

It is now straightforward to complete the proof. To prove (i) we observe that the number of triangles in $S$ is at most the number of triangles in $R_{K}$, which is at most 2304t, plus the number $k \ell$ of extra triangles added in the "twisting" step in constructing $S$ from $S_{0}$. A trivial bound for $\ell$ is given by the number of triangles in $M^{\prime \prime}$ which is at most 2304t. Combining this with (4.14) yields at most

$$
2304 t+k \ell \leq 2304 t+(2304 t+1) 3^{1152 t}(2304 t) \leq t^{2} \cdot 3^{1152 t+20}<2^{1858 t}
$$

triangles in $S$.

To prove (ii) we pull the curve $K$ across $S$ to $K_{2}$, across one triangle at a time. Since all vertices of $S$ are in the boundary $\partial S$, a legal order of triangles to pull across is easily found. Counting two elementary moves per triangle, this yields at most $2^{1858 t+1}$ elementary moves in total.

Remark. In the special case that the manifold $M$ is embedded in $\mathbb{R}^{3}$, as when we start with a standard knot complement, we can improve the bounds (i), (ii) of Theorem 4.1 to be polynomial in $n$. We sketch the idea. The knot $K$ has at most $2304 t$ edges in $M$. Using the embedding of the manifold $M$ in $\mathbb{R}^{3}$ we can find a regular projection of the knot to a knot diagram $\mathcal{D}$, and this has $O\left(n^{2}\right)$-crossings, as we show in Lemma 7.1 of $\S 7$. We can specify a curve $\alpha$ in the 1 -skeleton of $\partial R_{K}$ which is parallel to the core $K$ whose projection runs just to the right of $K$ in the projection plane. Choose an orientation of $K$. A longitude of a knot $K$ in 
$\mathbb{R}^{3}$ is characterized by having a linking number with $K$ equal to zero. The linking number is easily calculated from the projection. To each crossing of $K$ is associated a sign, and the sum of these signs, called the writhe of the knot, gives the linking number of a curve with $K$. To ensure that the linking number is zero, we alter the construction of $\alpha$. Near every overcrossing of $K$, we add a full twist of $\alpha$ around $K$. This twist is positive if the sign of the crossing is negative, and vice versa. The result is a knot $K_{2}$ in $\mathbb{R}^{3}$ that lies on the peripheral torus whose linking number with $K$ is zero. We can construct the associated annulus $S$ in $R_{K}$ with boundary components $K$ and $K_{2}$ as in the proof given for Theorem 4.1, and it has $O\left(n^{2}\right)$ triangles. The key point in this argument is that the writhe is bounded in terms of the number of crossings of $\mathcal{D}$, which is $O\left(n^{2}\right)$, and this gives a bound $k=O\left(n^{2}\right)$ in place of 4.14).

\section{ISOTOPIES OF CURVES ON A SURFACE}

In this section we examine isotopies between two $P L$ curves $\alpha$ and $\beta$ on an oriented triangulated surface $F$, and bound the number of elementary moves needed to move $\alpha$ to $\beta$. In the application, the surface will be the peripheral torus $\partial R_{K}$ of a knot $K, \alpha$ will be $K_{1}$, the boundary of a normal disk, isotopic on $\partial R_{K}$ to $\beta$, and $\beta$ will be a fixed longitudinal curve $K_{2}$, parallel to $K$ in its regular neighborhood $R_{K}$. We derive the results of this section for a general surface, since no extra work is needed.

To carry out this analysis we introduce a new notion, that of a basic curve. A basic curve is similar to a normal curve in having only a finite number of possible types in each triangle, but is more general, in that it allows curves that double back in a triangle, entering and leaving through the same edge. Starting with an arbitrary curve and isotoping it to become normal is a global operation, whereas altering a curve to become basic can be done simplex by simplex. Basic curves are piecewise-linear, with each component in a triangle having two or three edges. They seem well suited to computational modeling of isotopies of curves on surfaces. However they are not determined completely by a specification of the number of each of the six possible arc types in each triangle, so they lack the close relation of normal curves to an integer vector.

Our main result in this section is the following.

Theorem 5.1. Let $F$ be a triangulated orientable surface with $u$ triangles, and let $\alpha$ and $\beta$ be embedded isotopic PL curves on $F$, each having at most $s$ edges. Then there is an isotopy carrying $\beta$ to $\alpha$ using at most $2^{11}\left(s+u^{2}\right)^{4} u^{3}$ elementary moves.

We now define the class of basic arcs and curves. Let $E$ be a unit edge length equilateral triangle. Let $E^{\prime}$ be an equilateral triangle homothetic to $E$ with respect to its centroid, shrunk by a factor of 4; see Figure 9 A basic arc in $E$ falls into one of two types as in Figure 9 :

(a) Type 1. An arc running between the interiors of two distinct edges of $E$. This type of arc contains three edges. The first edge runs from the initial point towards the centroid of $E$ until it meets $E^{\prime}$. The third edge runs from the final point of the arc towards the centroid of $E$ until it meets $E^{\prime}$. The middle edge connects the outer two in $E^{\prime}$.

(b) Type 2. An arc with both of its endpoints on the interior of a single edge of $E$. This type of arc contains two edges, each of which makes an angle of $15^{\circ}$ with the edge. 


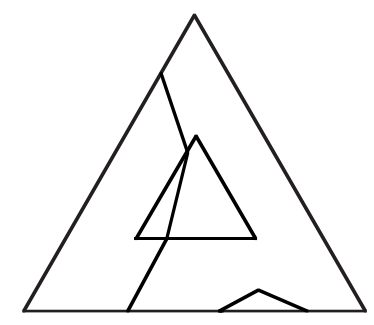

Figure 9 . Type 1 and type 2 basic arcs.

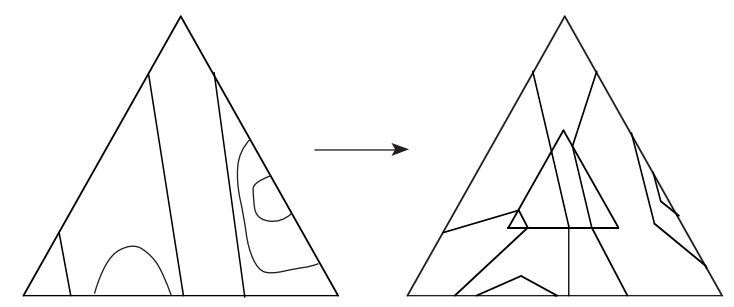

Figure 10. Any set of arcs can be isotoped to a set of basic arcs.

Basic arcs with non-linking endpoints are disjoint, since an arc of type 1 makes an angle larger than $\arctan (\sqrt{3} / 5) \approx 19^{\circ}$ with an edge of $E$, and arcs of type 2 make a strictly smaller angle of $15^{\circ}$ with the edges of $E$.

Now let $E_{1}$ be any triangle. A basic arc in $E_{1}$ is defined to be the image of a basic arc in $E$ under a linear map from $E$ to $E_{1}$.

A basic curve in a triangulated surface is a curve which meets each triangle in a union of basic arcs.

Lemma 5.1. Two basic arcs in a triangle with distinct endpoints intersect in either zero or one point. A collection of disjoint embedded arcs in a triangle can be isotoped, without moving endpoints, to a collection of disjoint basic arcs.

Proof. Two basic arcs with distinct endpoints intersect if and only if their endpoints link on the boundary of the triangle. Any two families of disjoint embedded arcs in a triangle are isotopic, rel boundary. (See Figure 10.)

A basic move of a basic curve in a triangulated surface is defined to be one of the following three types of curve isotopies, each of which produces a new basic curve, as in Figure 11

(a) Type 1. An isotopy that slides an arc of the curve that crosses an edge along that edge. This isotopy alters the curve in two adjacent triangles.

(b) Type 2. An isotopy that isotops an arc of the curve that crosses an edge of the triangulation across an adjacent vertex, and then isotops each arc of intersection with a triangle created or altered in this process to a basic arc.

(c) Type 3. An isotopy that slides an arc of the curve with both endpoints on one edge of a triangle $E$ across the 2-gon it forms with that edge. This results in the joining of two arcs in an adjacent triangle $E_{2}$. If the two arcs give a closed curve in $E_{2}$, the closed curve is removed. Otherwise, this new arc is isotoped in $E_{2}$ to a basic arc. 

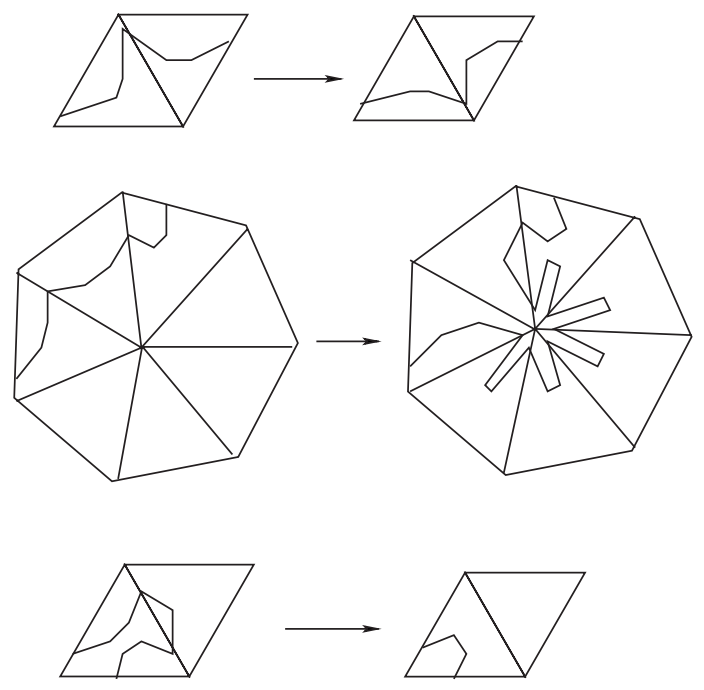

Figure 11. Basic move of types 1, 2 and 3.

We count the number of elementary moves needed to make a basic move. We first look in a single triangle.

Lemma 5.2. Let $\alpha=\alpha_{1} \cup \alpha_{2} \cup \ldots \cup \alpha_{n}$ and $\beta=\beta_{1} \cup \beta_{2} \cup \ldots \cup \beta_{n}$ each be a union of $n$ connected polygonal arcs in a triangle, with the endpoints of $\alpha_{i}$ coinciding with those of $\beta_{i}$, and with $a$ and $b$ edges respectively. Then $\alpha$ can be isotoped to $\beta$ in the triangle using at most $a+b$ type 2 elementary moves, and $2(a+b)$ elementary moves of both types.

Proof. We will count the number of required elementary moves in two cases. In each case we will isotop both $\alpha$ and $\beta$ to an intermediate curve $\gamma$, which stays close to the boundary of the triangle. To construct $\gamma$, we first pick $\theta$ to be a positive angle which is small enough so that for any vertex $v$ in $\alpha \cup \beta$ which lies on an edge of the triangle and any vertex $w$ in $\alpha \cup \beta$ which does not lie on that edge, the angle between that edge and the edge $v w$ is greater than $\theta$.

Given an $\operatorname{arc} \alpha_{i}$ of $\alpha$, we construct an arc $\gamma_{i}$ with the same endpoints, but with interior disjoint from $\alpha$ and containing just two edges. If $\alpha_{i}$ runs between distinct edges of the triangle, then it cuts off a subdisk containing a single vertex of the triangle. Construct $\gamma_{i}$ to consist of two edges, each of which makes an angle of $\theta$ with the edges going towards that vertex. By definition of $\theta, \gamma_{i}$ will be disjoint from the interiors of $\alpha_{i}$ and $\beta_{i}$. If $\alpha_{i}$ has both endpoints on a single edge of the triangle, then it cuts off a subdisk containing no vertices and a segment of one edge. Construct $\gamma_{i}$ to consist of two edges contained in this disk, each of which makes an angle of $\theta$ with its edge. Again, $\gamma_{i}$ will be disjoint from the interiors of $\alpha_{i}$ and $\beta_{i}$.

(1) $\alpha$ and $\beta$ are connected.

If $\alpha$ does not meet both sides of $\beta$, then the region between them can be triangulated with $a+b-2$ triangles, which can be used to isotop $\alpha$ to $\beta$ using $a+b-2$ type 2 elementary moves. If they intersect, then each can be isotoped to the disjoint curve $\gamma$ by using $a$ and $b$ type 2 elementary moves, 
respectively. It follows that we can always isotop $\alpha$ to $\beta$ using at most $a+b$ type 2 elementary moves, and $2(a+b)$ elementary moves of all types.

(2) $\alpha$ and $\beta$ are not connected.

We will construct an isotopy from $\alpha$ to $\beta$ through embedded curves. Let $a_{i}$ be the number of edges of $\alpha_{i}$, so that $\sum_{i=1}^{n} a_{i}=a$. Each arc $\alpha_{i}$ of $\alpha$ cuts off a sub-disk of the triangle containing zero or one vertex. An $\operatorname{arc}$ of $\alpha$ is said to be outermost if it cuts off a sub-disk which contains no other $\operatorname{arcs}$ of $\alpha$. Let $\alpha_{i}$ be outermost. Then $\gamma_{i}$ is disjoint from all the other arcs in $\alpha \cup \beta$ and meets $\alpha_{i} \cup \beta_{i}$ only in their endpoints. The number of edges of $\gamma_{i}$ is two, and $\gamma=\bigcup \gamma_{i}$ is embedded. We can isotop the outermost $\operatorname{arc} \alpha_{i}$ of $\alpha$ to the $\operatorname{arc} \gamma_{i}$ with the same endpoints without ever introducing any self-intersections in $\alpha$, since the other arcs of $\alpha$ lie on the other side of $\alpha_{i}$ from $\gamma_{i}$. This isotopy requires at most $a_{i}$ type 2 elementary moves, as in the previous case. We then repeat for an outermost arc $\alpha_{j}$ among the collection of $\operatorname{arcs}$ of $\alpha-\left\{\alpha_{i}\right\}$. This isotopy takes place between $\alpha_{j}$ and $\gamma_{j}$, and in particular never meets $\gamma_{i}$. Repeating for all $\operatorname{arcs}$ in $\alpha$, we can isotop the entire collection $\alpha$ to $\gamma$ using at most $\sum_{i=1}^{n} a_{i}=a$ type 2 elementary moves. Similarly, we can isotop $\beta$ to $\gamma$ using at most $b$ type 2 elementary moves. It follows that we can isotop $\alpha$ to $\beta$ using at most $a+b$ type 2 elementary moves.

The total number of elementary moves required in these isotopies is at most twice the number of type 2 elementary moves.

Define the length of a $P L$ curve transverse to the 1-skeleton of a triangulated surface to be the number of edges of the 1-skeleton that it crosses, counted with multiplicity.

Lemma 5.3. In a surface with a triangulation of degree at most $V$ at each vertex, a type 1 basic move can be realized by at most 28 elementary moves, a type 2 basic move by at most $6 \mathrm{~V}+24$ elementary moves, and a type 3 basic move by at most 22 elementary moves. In all cases $14 V$ elementary moves suffice.

Proof. We count the number of elementary moves required for each type of basic move.

Lemma 5.2 implies that a type 1 basic move requires at most 14 elementary moves in each of the two triangles meeting the edge along which the curve is isotoped.

The type 2 basic move can be done in the following sequence of steps. (See Figure 12, )

(1) Two type 2 elementary moves slide the arc in a neighborhood of an edge to pass through the adjacent vertex.

(2) Two type 2 elementary moves slide the curve to run along edges in a neighborhood of the adjacent vertex.

(3) $V-2$ type 2 elementary moves slide the arc over a neighborhood of the vertex in each adjacent triangle.

(4) At most seven type 2 elementary moves slide the arc to basic curves in each of the original two triangles, by Lemma 5.2 ,

(5) Two type 2 elementary moves slide the arc to basic curves in each of the $V-2$ triangles where new intersections were introduced.

Summing, this basic move requires less than $3 V+12$ type 2 elementary moves, and $6 V+24$ in total. 


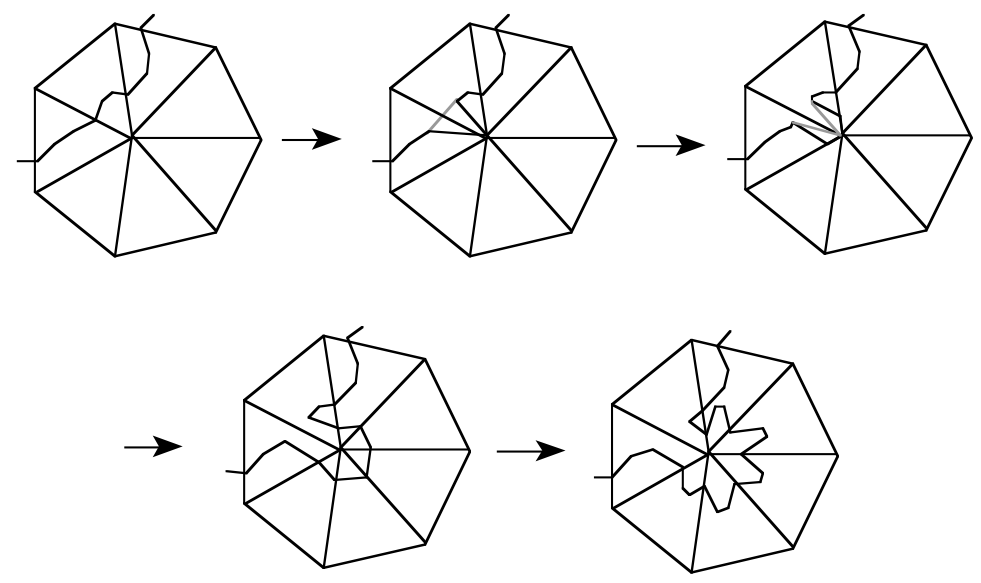

Figure 12. Elementary moves for a type 2 basic move.

The type 3 basic move requires

(1) One type 2 elementary move to slide the arc to coincide with a subarc of its adjacent edge.

(2) At most ten type 2 elementary moves slide the resulting arc to a basic curve with the same endpoints in the adjacent triangle, by an application of Lemma 5.2. This sums to at most 11 elementary moves of type 2 and 22 elementary moves of all types.

Since $V \geq 3$, in all cases $14 V$ elementary moves suffice.

If two basic curves are isotopic using only basic moves of type 1, we say that they are parallel basic curves.

Lemma 5.4. Let $\alpha$ and $\beta$ be two parallel basic curves with total length $\ell$. Then $\alpha$ can be isotoped to coincide with $\beta$ by $\ell / 2$ basic moves of type 1 . Similarly, $\alpha$ can be isotoped across $\beta$ by $\ell / 2$ basic moves of type 1 .

Proof. Each of $\alpha$ and $\beta$ has length $\ell / 2$ and they intersect each triangle of the surface in pairs of parallel basic arcs. Using $\ell / 2$ basic moves of type 1 , we can either move the intersections of $\alpha$ along each edge to coincide with the intersection of $\beta$ with that edge, or move the intersections past to the other side of $\beta$.

Let $\alpha$ and $\beta$ be intersecting simple closed curves in general position on a surface $F$. A 2-gon is a subdisk of $F$ whose boundary consists of one subarc of each of $\alpha$ and $\beta$. A 2-gon is innermost if no arc of $\alpha$ or $\beta$ meets its interior. In some cases, it can be shown that curves with excess intersection create innermost 2-gons. This is analyzed in some generality in [14. The following is Lemma 3.1 of [14].

Lemma 5.5. Let $F$ be a compact orientable surface and let $\alpha$ and $\beta$ be isotopic simple closed curves on $F$, intersecting transversely. Then either $\alpha$ and $\beta$ are disjoint or there is an innermost 2-gon on $F$ bounded by a sub-arc of each of $\alpha$ and $\beta$.

Proof. The lift of $\alpha$ to the universal cover of $F$ is a family of disjoint lines $\tilde{\alpha}$, and the lift of $\beta$ is a similar family $\tilde{\beta}$. Assume $\alpha$ and $\beta$ intersect. Then a lift $\alpha_{1}$ of $\alpha$ 
and a lift $\beta_{1}$ of $\beta$ also intersect. The curves $\alpha_{1}$ and $\beta_{1}$ are isotopic to disjoint lines by a lift of an isotopy making $\alpha$ and $\beta$ disjoint. The fundamental group of $F$ acts on the universal cover, and $\alpha_{1}$ and $\beta_{1}$ have a common cyclic stabilizer. It follows that they intersect infinitely often. An arc of each between adjacent crossing points cuts off a 2-gon in the universal cover. A finite number of arcs of $\tilde{\alpha} \cup \tilde{\beta}$ meet this 2 -gon. Each such arc is disjoint from either $\tilde{\alpha}$ or $\tilde{\beta}$, so that it cuts off a smaller 2 -gon inside the previous one. We can pass to an innermost 2-gon whose interior meets no arc of $\tilde{\alpha} \cup \tilde{\beta}$. This 2-gon is disjoint from any of its translates, and must project 1-1 down to $F$ under the covering projection. Accordingly, its image in $F$ is an innermost 2-gon.

Recall that the length of a curve is the number of edges it crosses.

Lemma 5.6. Let $F$ be an orientable triangulated surface with $u$ triangles, and let $\alpha$ and $\beta$ be isotopic simple basic curves on $F$ intersecting transversely. Let $l$ be the length of $\alpha \cup \beta$, and let $V$ be the maximal degree of a vertex in $F$. There is an isotopy carrying $\beta$ to $\alpha$ via no more than $l^{4} u V$ basic moves.

Proof. We treat cases. Note that $V \geq 3, l \geq 2$.

Case 1: $\alpha$ and $\beta$ are disjoint.

Then $\alpha$ and $\beta$ bound a subsurface of $F$ homeomorphic to an annulus. We will perform a series of basic moves which isotop the curves to coincide.

The triangulation of $F$ induces a cellulation of the annulus. The cells are subdisks of triangles, bounded by arcs in $\alpha \cup \beta$ and a subset of the 1-skeleton of $F$. If there are any vertices of $F$ in this cellulation, we can slide $\alpha$ or $\beta$ over a vertex by a basic move of type 2 . Repeating at most $u$ times, we eliminate all vertices in the annulus. The length of $\alpha \cup \beta$ is increased by at most $V-2$ each time we do a basic move, so the final curves have total length at most $l+u(V-2)=l+u V-2 u$.

The annulus between $\alpha$ and $\beta$ is now cut by the edges of $F$ into a collection of cells. Since $\alpha$ and $\beta$ are disjoint, and each cell boundary contains an edge lying on the 1-skeleton between each edge lying on $\alpha \cup \beta$, all cells are even sided. An Euler characteristic calculation shows that either all cells are 4-gons, or there is a 2 -gon cell in the annulus, bounded by a basic arc of type 2 and an arc on an edge of the triangulation.

If there is a cell which is a 2-gon, we can perform a basic move of type 3 which slides an arc of $\alpha$ or $\beta$ across that 2-gon. This decreases the length of $\alpha \cup \beta$ by two. After repeating at most $(l+u V-2 u) / 2$ times, we eliminate all 2-gon cells from the annulus, and arrive at a cellulation in which all cells are 4-gons. When all cells are 4-gons, the two boundary components of the annulus are parallel basic curves, each of length at most $(l+u V-2 u) / 2$. Using at most $(l+u V-2 u) / 2$ basic moves of type 1 , we can make the two curves coincide. by

The total number of basic moves required in the three steps above is bounded

$$
u+(l+u V-2 u) / 2+(l+u V-2 u) / 2=l+u V .
$$

Case 2: $\alpha$ and $\beta$ intersect.

Two basic arcs in a triangle with distinct endpoints intersect at most once, so the total number of intersections of $\alpha$ and $\beta$ is at most $l^{2} / 4$. Lemma 5.5 gives the existence of an innermost disk $D_{1}$ on the surface, bounded by subarcs $\alpha_{1}$ of $\alpha$ and $\beta_{1}$ of $\beta$. The 1 -skeleton of $F$ induces a cellulation of $D_{1}$. The cells are bounded by $\operatorname{arcs}$ in $\alpha_{1} \cup \beta_{1}$ and $\operatorname{arcs}$ in the 1 -skeleton of $F$. If $D_{1}$ contains a vertex of $F$, we can 
isotop $\alpha_{1}$ or $\beta_{1}$ over a vertex by a basic move of type 2 , keeping $\alpha \cup \beta$ embedded. The number of vertices is bounded above by $u$, so repeating at most $u$ times, we eliminate all vertices in $D_{1}$, using at most $u$ basic moves of type 2 . The length of $\alpha_{1} \cup \beta_{1}$ is increased by at most $V-2$ by each basic move, so $\alpha$ and $\beta$ are isotoped to curves with total length less than $l+u(V-2)=l+u V-2 u$.

Since $\alpha_{1}$ and $\beta_{1}$ meet at exactly two points in the boundary of $D_{1}$, there are at most two cells in $D_{1}$ with an odd number of sides. An Euler characteristic calculation shows that one of three cases occurs:

Case 1. all of $D_{1}$ is contained in a single triangle, or

Case 2 . there is a 2 -gon cell in $D_{1}$, with one edge on $\alpha$ or $\beta$ and one edge on the 1-skeleton, or

Case 3. all cells of $D_{1}$ are 4-gons, other than two 3-gons containing points of $\alpha_{1} \cap \beta_{1}$.

The first case is impossible because basic arcs don't intersect in more than one point in a single triangle. A 2 -gon cell in $D_{1}$ gives a basic arc of type 2 . We can use this to perform a basic move of type 3 , which slides an arc of $\alpha_{1}$ or $\beta_{1}$ across a 2-gon. This decreases the length of $\alpha_{1} \cup \beta_{1}$ by two, and thus can be repeated at most $(l+u V-2 u) / 2$ times. This leaves us in Case 3. We can then isotop $\beta_{1}$ across $\alpha_{1}$, using at most $(l+u V-2 u) / 2$ basic moves of type 1 . We have then eliminated the innermost 2-gon $D_{1}$, reducing the number of intersection points of $\alpha$ and $\beta$ by two, while using at most $l+u V$ basic moves.

The length of $\alpha \cup \beta$ is now bounded by the larger value $l+u V$. If $\alpha$ and $\beta$ still intersect, we repeat with another innermost disk. Eliminating this disk requires at most $(l+u V)+u V=l+2 u V$ basic moves. Repeating at most $l^{2} / 4$ times we eliminate all intersections and arrive at disjoint curves. Summing the resulting series, which has at most $l^{2} / 4$ terms, gives a bound of

$$
\begin{aligned}
l+u V+(l+2 u V)+\cdots+\left(l+\left(l^{2} / 4\right) u V\right) & \leq l^{3} / 4+\frac{l^{2}}{8}\left(\frac{l^{2}}{4}-1\right) u V \\
& \leq l^{3} / 4+l^{4} u V / 32
\end{aligned}
$$

basic moves. With the curves now disjoint, and having length less than $l+\frac{l^{2}}{4} u V$, Case 1 isotops them to coincide using at most $l+\frac{l^{2}}{4} u V+u V$ basic moves. The total number of basic moves used in the isotopy is therefore less than

$$
l^{3} / 4+l^{4} u V / 32+l+\frac{l^{2}}{4} u V+u V
$$

Since $l \geq 2$ we have

$$
\begin{aligned}
l^{4} u V & \geq l^{4} u V / 32+3 l^{3} u V / 4 \\
& \geq l^{4} u V / 32+l^{3} u V / 4+l^{2} u V \\
& \geq l^{4} u V / 32+l^{3} / 4+l+l^{2} u V / 4+3 l^{2} u V / 4 \\
& \geq l^{4} u V / 32+l^{3} / 4+l+l^{2} u V / 4+u V,
\end{aligned}
$$

so the sum is less than $l^{4} u V$ as claimed.

Lemma 5.7. Let $F$ be an orientable triangulated surface with $u$ triangles, and let $\alpha$ and $\beta$ be isotopic basic curves on $F$ in general position. Let $l$ be the total length of $\alpha \cup \beta$. Then there is an isotopy carrying $\beta$ to $\alpha$ via no more than $14 l^{4} u^{3}$ elementary moves. 
Proof. The required number of basic moves is at most $l^{4} u V$, and each basic move requires at most $14 \mathrm{~V}$ elementary moves, so the total number of elementary moves required is less than $14 l^{4} u V^{2}$. We know $V \leq u$ so that $14 l^{4} u^{3}$ gives an upper bound on the number of elementary moves.

We now give the proof of the main result of this section.

Proof of Theorem [5.1. We begin by isotoping $\alpha$ to a basic curve. First we perturb $\alpha$ to miss the zero-skeleton of the triangulation. There are at most $u$ vertices, and each has at most $V$ edges coming into it, where $V$ is the maximal degree of the triangulation of the surface. We can perturb $\alpha$ near any vertex which it passes through to a new $P L$ curve that crosses at most $V / 2$ edges near that vertex. This requires a total of at most $u(V / 2+1)$ type 2 elementary moves, and $u V+2$ elementary moves of both types. The number of edges on the curve has now been increased to at most $s+u(V / 2+1)<s+u V$.

Next we isotop the intersection of $\alpha$ with each triangle to a union of basic arcs. This can increase the number of edges by at most a factor of three to $3(s+u V)$ and requires less than $4(s+U V)$ elementary moves by Lemma 5.2 We can do similar moves on $\beta$, resulting in new curves $\alpha$ and $\beta$ which are transversely intersecting basic curves with a total of at most $6(s+u V)$ edges. Note that the length of a basic curve is between one third and one half of the number of edges it contains, therefore $\alpha \cup \beta$ has length at most $3(s+u V)$. We now apply Lemma 5.7 isotoping the curves together using at most $14[3(s+u V)]^{4} u^{3}$ elementary moves. We can bound $V$ by $u$, obtaining a bound of at most

$$
\left(u^{2}+2\right)+4\left(s+u^{2}\right)+1134\left[\left(s+u^{2}\right)\right]^{4} u^{3}<2^{11}\left(s+u^{2}\right)^{4} u^{3}
$$

elementary moves.

\section{Proof of Theorem 1.2}

We combine the results of $\S 2-\S 5$ to prove Theorem 1.2

Proof of Theorem [1.2, We barycentrically subdivide $M$ twice and construct a new triangulated manifold $M_{K}=M-R_{K}$, by removing the interior of a regular neighborhood $R_{K}$ of $K$. The boundary of $M_{K}$ consists of a subdivision of the original boundary $\partial M$ plus a 2 -torus $\partial R_{K}$, the peripheral torus. The manifolds $M_{K}$ and $R_{K}$ contain between them $576 t$ tetrahedra. As described in $\S 3$, we deform the original knot $K$ across an annulus by elementary moves to a longitude $K_{1}$ on the peripheral 2-torus $\partial R_{K}$. Then we deform $K_{1}$ on the peripheral torus by elementary moves to the boundary $K_{2}$ of the embedded normal disk given by Haken's Theorem 3.1. Finally we deform the curve $K_{2}$ across this disk using elementary moves to a triangle.

We count elementary moves. By Lemma 3.4 the normal disk with $K_{2}$ as boundary contains at most $2^{8 \cdot(576 t)+6}=2^{4608 t+6}$ triangles, and the number of edges $\left|K_{2}\right|$ of $K_{2}$ is at most

$$
\left|K_{2}\right| \leq 2^{4608 t+7}<2^{4609 t}=s_{2} .
$$

Also by Lemma 3.4 it takes at most $2^{4608 t+7}$ elementary moves to transform $K_{2}$ to a triangle.

By Theorem 4.1 the longitude $K_{1}$ consists of at most

$$
\left|K_{1}\right| \leq 2^{1858(576 t)+1}<2^{1070209 t}=s_{1}
$$


edges, and the isotopy from $K$ to $K_{1}$ requires at most the same number of elementary moves.

It remains to bound the number of elementary moves needed to carry $K_{1}$ to $K_{2}$. Note that $K_{1}$ is isotopic to $K_{2}$ if properly oriented. Theorem 5.1 applies with $s=\max \left(s_{1}, s_{2}\right)=s_{1}$ to yield a bound of at most $2^{11}\left(s+u^{2}\right)^{4} u^{3}$ elementary moves taking $K_{1}$ to $K_{2}$, where $u$ is the number of triangles on the peripheral torus. Since $u \leq 2(576) t<2^{11} t$, Theorem 5.1 gives an upper bound of $2^{10^{7} t-1}$ for the number of elementary moves taking $K_{1}$ to $K_{2}$. For the three steps together we obtain a bound of $2^{4609 t}+2^{1070209 t}+2^{10^{7} t-1}<2^{10^{7} t}$.

Remark. The assumption $K \subseteq \operatorname{int}(M)$ was used to allow the peripheral torus $R_{K}$ to be constructed by two barycentric subdivisions so that $\partial R_{K}$ is disjoint from $\partial M$. For the general case that $K \subseteq M$, we could proceed by barycentrically subdividing $M$ twice, then isotoping $K$ off the boundary $\partial M$ to a "parallel" knot $K^{\prime \prime}$ contained strictly in the interior of $M^{\prime \prime}$, using $O(t)$ elementary moves. Now Theorem 1.2 applies to $K^{\prime \prime}$ in $\left(M^{\prime \prime}, \partial M^{\prime \prime}\right)$.

\section{REIDEMEISTER MOVES AND ELEMENTARY MOVES IN $\mathbb{R}^{3}$}

The remainder of the paper is concerned with 3-manifolds embedded in $\mathbb{R}^{3}$, and is devoted to proving the Reidemeister move bound of Theorem 1.1.

We outline the proof of Theorem 1.1. We have a knot diagram $\mathcal{D}$ with $n$ vertices. We use it to construct a triangulated $P L$ manifold $M$ embedded in $\mathbb{R}^{3}$ which contains a knot $K$ in its 1 -skeleton whose projection on the $x y$-plane in $\mathbb{R}^{3}$ is the knot diagram $\mathcal{D}$. In $\S 8$ we show that such a manifold $M$ can be constructed using $O(n)$ tetrahedra. Now Theorem 1.2 shows that we can isotop $K$ to a trivial knot using $2^{c_{3} n}$ elementary moves. We project these elementary moves down onto the $x y$-plane and obtain a sequence of Reidemeister moves. The proof is completed by bounding the number of Reidemeister moves in terms of elementary moves, which we do below.

In the rest of this section we describe Reidemeister moves and relate them to elementary moves. We recall basic facts about knot and link diagrams.

A projection of a link in $\mathbb{R}^{3}$ into a plane is regular if all crossings are in general position, and at most 2 edges intersect the pre-image of any point. Corresponding to a regular projection of a link is a link diagram. This is a labeled planar graph having the following properties:

(i) Any connected component of $\mathcal{D}$ with no vertices is an (isolated) loop.

(ii) Each vertex has exactly four adjacent edges, with two edges labeled "undercrossing" and two labeled "overcrossing" at the vertex.

(iii) The labeling prescribes a cyclic ordering of edges at each vertex, and "overcrossing edges" are adjacent to "undercrossing edges" in this ordering.

Conversely, for any labeled planar graph $\mathcal{D}$ satisfying (i)-(iii) there exists an unoriented link $L$ having projection $\mathcal{D}$. We note that the cyclic ordering of vertices in (iii) actually specifies a planar embedding of the graph $\mathcal{D}$, since it determines the faces of the planar embedding.

A link component in a link diagram $\mathcal{D}$ is obtained by tracing a closed path of edges such that each pair of consecutive edges match at their common vertex, either both overcrossing or both undercrossing. The order of $\mathcal{D}$ is the number of components it contains. A link diagram component of $\mathcal{D}$ is a connected component 


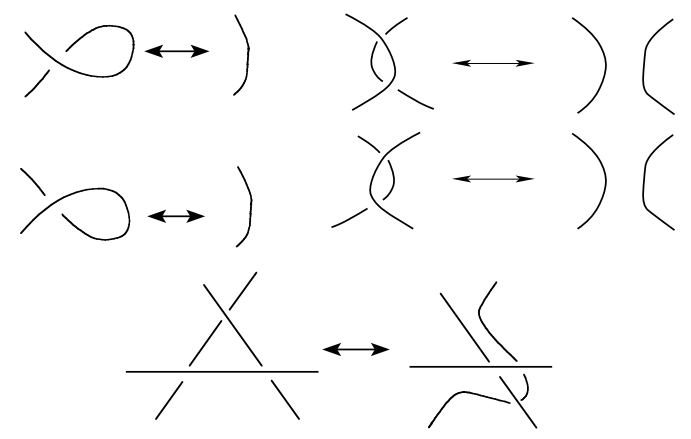

Figure 13. Reidemeister moves of types I, II and III.

of the underlying graph; it can be the union of several link components. The crossing measure $|\mathcal{D}|$ of a link diagram is

$$
|\mathcal{D}|=\# \text { (vertices })+\#(\text { link diagram components })-1 \text {. }
$$

A knot diagram is a link diagram that consists of one link component. A trivial knot diagram is a knot diagram that has no vertices. If $\mathcal{D}$ is a knot diagram, then $|\mathcal{D}|$ is the crossing number of the knot projection.

Lemma 7.1. If a polygonal link $L$ has regular projection $\mathcal{D}$, then

$$
|\mathcal{D}| \leq|L|^{2}
$$

Proof. The number of vertices $|L|$ is at least as large as the number of distinct edges it contains. Since edges project to edges, each pair of edges produces at most one crossing. There are less than $|L|^{2}$ pairs. Furthermore edges that project into different connected components of $|\mathcal{D}|$ cannot produce any crossings. Thus the number of non-intersecting pairs of edges is at least \#(components of $\mathcal{D})-1$, so (7.18) follows.

The bound (17.18) is the correct order of magnitude, because one can easily construct a sequence of knots $K$ with $|K| \rightarrow \infty$, which have the property that $|\mathcal{D}|>$ $\frac{1}{2}|K|^{2}$. There is no upper bound for $|L|$ in terms of $|\mathcal{D}|$, but there does exist a link $L$ with a projection having diagram $\mathcal{D}$, with $|L| \leq c|\mathcal{D}|$; see [12, Lemma 5.1].

Reidemeister moves are local moves on link diagrams that are analogous to elementary moves. They convert a link diagram $\mathcal{D}$ to another link diagram $\mathcal{D}^{\prime}$ by a local change in its structure. For unoriented links there are three kinds of Reidemeister moves, examples of which are pictured in Figure 13, see one of [21, p. 48], [1], 6], [25]. (For oriented links there are more Reidemeister moves; see $\mathrm{Wu}$ [33].)

We call two knot diagrams $\mathcal{D}_{1}$ and $\mathcal{D}_{2}$ equivalent if and only if one can be converted into the other by a finite sequence of Reidemeister moves. This relation corresponds to knot equivalence: Knots $K_{1}$ and $K_{2}$ have knot diagrams $\mathcal{D}_{1}$ and $\mathcal{D}_{2}$ that are equivalent if and only if $\mathcal{D}_{1}$ and $\mathcal{D}_{2}$ are equivalent.

Reidemeister moves on a link diagram $\mathcal{D}$ are related to elementary moves on a link $L$ whose projection is $\mathcal{D}$, but the correspondence of moves is not one-to-one.

Lemma 7.2. Let $L$ be a polygonal link and suppose that it has diagram $\mathcal{D}$ as regular projection on a plane. Let $L^{\prime}$ be a link derived from $L$ by an elementary move of type (2) or $\left(2^{\prime}\right)$, and suppose that it has regular projection $\mathcal{D}^{\prime}$ on the same plane. 
Then there is a sequence of at most $2|L|+2|\mathcal{D}|$ Reidemeister moves that converts $\mathcal{D}$ to $\mathcal{D}^{\prime}$.

Proof. As an edge is moved affinely in $\mathbb{R}^{3}$ with one end fixed, its projection intersects the projection of any vertex of the polygonal link $L$ in at most one point. Therefore it can generate at most one type I or type II Reidemeister move involving the projection of that vertex. The number of vertices is bounded by $|L|$. Similarly, each pair of crossing edges in $\mathcal{D}$ can produce at most one type III Reidemeister move. Since two edges are moved in a type $(2)$ or $\left(2^{\prime}\right)$ elementary move, this yields at most $2|L|+2|\mathcal{D}|$ Reidemeister moves.

The bound of Lemma 7.2 is nearly best possible.

We use these lemmas to prove a bound for Reidemeister moves in terms of elementary moves.

Theorem 7.1. Let $L$ and $L^{\prime}$ be polygonal links each consisting of at most $n$ edges and suppose that $\mathcal{D}$ and $\mathcal{D}^{\prime}$ are link diagrams obtained by regular projection of $L$ and $L^{\prime}$ on a fixed plane. If $L$ can be transformed to $L^{\prime}$ using $k$ elementary moves, then $\mathcal{D}$ can be transformed to $\mathcal{D}^{\prime}$ using at most $2 k\left(n+\frac{1}{2} k+1\right)^{2}$ Reidemeister moves.

Proof. Let $L=L_{0}, L_{1}, L_{2}, \ldots, L_{k}=L^{\prime}$ be the set of intermediate links occurring in the sequence of elementary moves, and let $\mathcal{D}_{i}$ denote the projection of $L_{i}$ on the fixed plane. We may assume that all projections are regular, by applying a transversality argument and infinitesimally moving the plane of projection [6. Prop. 1.12]. Since an elementary move changes the number of vertices by at most one, and $\left|L_{0}\right|,\left|L_{k}\right| \leq n$, we have, for $1 \leq i \leq k$, that

$$
\left|L_{i}\right| \leq \min (n+i, n+k-i) \leq n+\frac{1}{2} k .
$$

Lemma 7.1 gives

$$
\left|\mathcal{D}_{i}\right| \leq\left|L_{i}\right|^{2} \leq\left(n+\frac{1}{2} k\right)^{2}=n^{2}+n k+\frac{k^{2}}{4}
$$

Thus we obtain

$$
2\left|L_{i}\right|+2\left|\mathcal{D}_{i}\right| \leq 2\left(n+\frac{1}{2} k+1\right)^{2} .
$$

Applying Lemma 7.2 to each $L_{i}$ separately yields the upper bound $2 k\left(n+\frac{1}{2} k+1\right)^{2}$.

\section{TRIANGUlation OF KNOT COMPLEMENTS IN $\mathbb{R}^{3}$}

In this section we construct a triangulated 3-manifold $M_{K}$ which is a knot complement in a ball embedded in $\mathbb{R}^{3}$. That is, $M_{K}=S^{3}-\left(R_{K} \cup B\right)$, in which $R_{K}$ is an open regular neighborhood of the knot $K, B$ is an open ball containing the point at infinity in its interior, and $\bar{R}_{K} \cap \bar{B}=\emptyset$. The manifold $M_{K}$ has boundary $\partial M_{K}=\partial R_{K} \cup \partial B$ which consists of two connected components, which are topologically a 2-sphere and a torus. As in $\S 2$, we call such a manifold $M_{K}$ a truncated knot complement.

We carry out this construction more generally for link diagrams, as it requires no extra work. Given a link diagram $\mathcal{L}$ with crossing measure $n$, we construct a triangulated manifold $M$ in $\mathbb{R}^{3}$ which contains $O(n)$ tetrahedra and has a link $L$ in its 1 -skeleton which has a regular projection that gives the link diagram $\mathcal{L}$. 
Theorem 8.1. Given a link diagram $\mathcal{L}$ of crossing measure $n$, one can construct a triangulated convex polyhedron $P$ in $\mathbb{R}^{3}$ such that:

(i) The triangulation contains at most 840 n tetrahedra.

(ii) Every vertex in the triangulation is a lattice point $(x, y, z) \in \mathbb{Z}^{3}$, with $0 \leq x \leq 30 n, 0 \leq y<30 n$ and $-6 \leq z \leq 6$.

(iii) There is a link $\mathcal{L}$ embedded in the 1-skeleton of the triangulation which lies entirely in the interior of $P$, and whose orthogonal projection on the plane $z=0$ is regular and is a link diagram isomorphic to $\mathcal{L}$.

Proof. The link diagram $\mathcal{L}$ comes with a topological planar embedding which determines the faces. We begin by adding extra vertices to $\mathcal{L}$. To each edge or non-isolated loop we add two new vertices of degree 2 , which splits it into three edges. To each isolated loop we add three new vertices of degree 2, making it an isolated triangle. The resulting labeled graph $\mathcal{G}$ is still planar, has no loops or multiple edges, and has at most $5 n$ vertices. (The worst case consists of several disjoint single crossing projections.) Let $m$ denote the number of vertices of $\mathcal{G}$, and call the $m-n$ vertices added special vertices. Note that each vertex of the original graph has associated to it four distinct special vertices.

Using the topological planar embedding of $\mathcal{G}$, we next add extra edges to triangulate each face of $\mathcal{G}$, obtaining a topologically embedded triangulated planar graph $\mathcal{G}^{\prime}$. The graph $\mathcal{G}^{\prime}$ has $m$ vertices and $2 m-4$ bounded triangular faces, and the unbounded face is also a triangle. We next encase the graph $\mathcal{G}^{\prime}$ in a triangulated planar graph $\mathcal{H}^{\prime}$ with $m+3$ vertices and $2 m+2$ bounded triangular faces, by framing the outside triangular face with a larger triangle and then adding 6 edges to subdivide the resulting concentric region into 6 triangles, obtaining $\mathcal{G}^{\prime \prime}$. See Figure 14

Our next goal is to replace the topological embedding of $\mathcal{G}^{\prime \prime}$ in the plane with a straight-line embedding. In 1952 Fary showed that straight-line embeddings always exist, and a recent result of de Fraysseix, Pach and Pollack [7] states that the vertices can be chosen to lie in a small rectangular grid of integer lattice points. Their results imply that there exists a planar embedding of $\mathcal{H}^{\prime}$ whose vertices $V\left(\mathcal{H}^{\prime}\right)$ lie in the plane $z=-1$, are contained in the grid

$$
\{(x, y,-1): 0 \leq x, y \leq 10 n-1 ; \quad x, y \in \mathbb{Z}\},
$$

and all edges of the graph $\mathcal{H}^{\prime}$ are straight line segments.

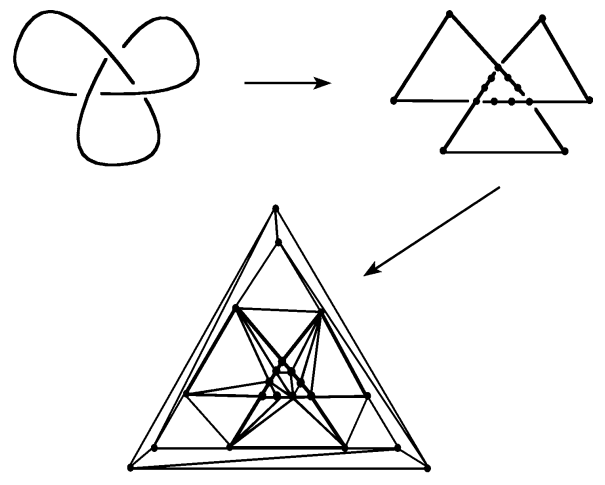

FiguRE 14. Triangulating a knot projection. 
Next we make an identical copy $\mathcal{H}^{\prime \prime}$ of $\mathcal{H}^{\prime}$ on the plane $z=1$ with $\mathcal{H}^{\prime \prime}=$ $\mathcal{H}^{\prime}+(0,0,2)$, and vertex set $V\left(\mathcal{H}^{\prime \prime}\right)=V\left(\mathcal{H}^{\prime}\right)+(0,0,2)$. We now consider the polyhedron $P^{\prime}$ which is the convex hull of $V\left(\mathcal{H}^{\prime}\right)$ and $V\left(\mathcal{H}^{\prime \prime}\right)$. It is a triangular prism, because the outside face of $\mathcal{H}^{\prime}$ is a triangle. We add $m_{1}$ vertical edges connecting each vertex $\mathbf{v} \in V\left(\mathcal{H}^{\prime}\right)$ to its copy $\mathbf{v}+(0,0,2) \in V\left(\mathcal{H}^{\prime \prime}\right)$. Let $\mathcal{E}$ denote these edges, together with all the edges in $\mathcal{H}^{\prime}$ and $\mathcal{H}^{\prime \prime}$. Using these edges, the polyhedron $P^{\prime}$ decomposes into $2 m+2$ triangular prisms $\left\{Q_{j}: 1 \leq j \leq 2 m-4\right\}$, with the top and bottom faces of each being congruent triangular faces of $\mathcal{H}^{\prime}$ and $\mathcal{H}^{\prime \prime}$.

We next triangulate $P^{\prime}$ by dissecting each of the $2 m+2$ triangular prisms into 14 tetrahedra, as follows. We subdivide each vertical rectangular face into four triangles using its diagonals. Then we cone each rectangular face to the centroid of $P^{\prime}$, and note that the centroid lies in the plane $z=0$. We add four new vertices to each prism, one on each rectangular face and one in the center. The point of this subdivision is that the triangulations of adjacent prisms are compatible. Note also that all new vertices added lie in the lattice $\frac{1}{3} \mathbb{Z}^{3}$.

Let $\mathcal{E}^{\prime}$ denote all the edges in the union of these triangulated prisms. We identify the link diagram $\mathcal{G}$ with the graph $\mathcal{G}$ embedded in $\mathcal{H}^{\prime}$. We next observe that there is a polygonal link $L$ embedded in the edge set $\mathcal{E}^{\prime}$ whose projection is the link diagram $\mathcal{L}$. We insist that any edge in $\mathcal{D}$ that runs to an undercrossing have its vertex which is an undercrossing be in the plane $z=-1$, while any edge that runs to an overcrossing have the overcrossing vertex in the plane $z=1$. Each such edge goes from one of the $n$ original vertices of $\mathcal{L}$ to a special vertex adjacent to that vertex. Edges that do not meet vertices labeled overcrossing or undercrossing are assigned to the $z=-1$ plane. The edge corresponding to an edge running from the $z=-1$ to the $z=1$ plane is contained in one of the diagonals added to a prism. The resulting embedding $L$ in $P^{\prime}$ has a regular orthogonal projection onto the $(x, y)$-plane, since no edges are vertical and only transverse double points occur as singularities in the projection. Note that no edge of $L$ touches the outer triangle of $\mathcal{H}^{\prime}$ because all edges project to the graph $\mathcal{G}^{\prime}$ contained strictly inside $\mathcal{H}^{\prime}$. This will be important for property (iii).

The knot edges lie in the upper and lower boundary of $P^{\prime}$, but do not touch its sides. To have them not touch the boundary we take two additional copies of $P^{\prime}$ and glue one to its top, along the plane $z=1$, and one to its bottom, along the plane $z=-1$. The knot now lies entirely in the interior of the resulting polyhedron $P^{\prime \prime}$. All the vertices added in this construction lie in $\frac{1}{3} \mathbb{Z}^{3}$. The total number of tetrahedra used in triangulating $P^{\prime \prime}$ is $84(m+1)$, which is at most $420(n+1)$, or at most $840 n$ for $n \geq 1$.

Now triple all coordinates $(x, y, z) \rightarrow(3 x, 3 y, 3 z)$ to obtain a triangulated polytope $P=3 P^{\prime \prime}$ with integer lattice point vertices, with a triangulation that satisfies (i)-(iii).

Remark. (1) The construction in Theorem 8.1 can be effectively computed in time $O(n \log n)$ using the Hopcroft-Tarjan planarity algorithms [16] and the embedding algorithm of de Fraysseix, Pach and Pollack [7, Section 4].

(2) One can ask whether a polygonal knot $K$ in $\mathbb{R}^{3}$ that consists of $n$ line segments can be embedded in the 1-skeleton of some triangulated polyhedron that uses $O(n)$ tetrahedra. We do not know if this can always be done. There exist polygonal knots whose planar projections in any direction have at least $\Omega\left(n^{2}\right)$ crossings. Avis 
and El Gindy [3] showed that a set of $n$ points in general position in $\mathbb{R}^{3}$ can be embedded as vertices of a triangulation of their convex hull using $O(n)$ tetrahedra, but that in the general case $\Omega\left(n^{2}\right)$ tetrahedra are sometimes needed.

\section{Proof of Theorem 1.1}

We combine the earlier results to prove Theorem 1.1 with an explicit bound on the number of Reidemeister moves required to isotop an unknot to the trivial projection.

Proof of Theorem 1.1. Applying Theorem 8.1 to the knot diagram $\mathcal{D}$ yields a triangulated convex polyhedron $M$ embedded in $\mathbb{R}^{3}$ with $t$ tetrahedra, where

$$
t \leq 840 n
$$

and with a knot $K$ embedded in the 1-skeleton of the interior of $M$ that projects to $\mathcal{D}$. The hypotheses of Theorem 1.2 are satisfied for $M$, hence there is a sequence of at most $2^{c_{2} t}$ elementary moves transforming $K$ to the trivial knot in $M$, with $c_{2}=10^{7}$. Now Theorem 7.1 gives a bound of at most

$$
2^{c_{2} t}\left(6 \cdot 840 n+\frac{1}{2} \cdot 2^{c_{2} t}+1\right)^{2}
$$

Reidemeister moves, since $K$ contains at most $6 \cdot 840 n$ line segments.

The bound (9.21) yields the desired bound of the form $2^{c_{1} n}$ for an explicit constant $c_{1}$. Using the value $c_{2}=10^{7}$ from the proof of Theorem 1.2 one finds that $c_{1}=10^{11}$ suffices.

\section{REFERENCES}

[1] C. Adams, The Knot Book. An elementary introduction to the mathematical theory of knots, W. H. Freeman, New York, 1994. MR 94m:57007

[2] J. W. Alexander and G. B. Briggs, On types of knotted curves, Ann. Math., 28 (1926/27), $562-586$.

[3] D. Avis and H. El Gindy, Triangulating point sets in space, Disc. \& Comp. Geom., 2 (1987), 99-111. MR 88h:68082

[4] J. Birman and M.D. Hirsch, Recognizing the unknot, Geom. Topol., 2 (1998), 175-220. MR 2000a:57005

[5] J. Birman and W. Menasco, Studying links via closed braids V: The unlink, Trans. AMS, 329 (1992), 585-606. MR 92g:57010b

[6] G. Burde and H. Zieschang, Knots, de Gruyter, 1985. MR 87b:57004

[7] H. de Fraysseix, J. Pach and R. Pollack, How to draw a planar graph on a grid, Combinatorica, 10 (1990), 41-51. MR 91g:05123

[8] S. Galatolo, On a problem in effective knot theory, Rendiconti dell Accademia dei Lincei, 9 (1999), no. 4, 299-306. MR 2000j:57010

[9] L. Goeritz, Bemerkungen zur knotentheorie, Abh. Math. Sem. Univ. Hamburg, 10 (1934), 201-210.

[10] W. Haken, Theorie der Normalflachen, ein Isotopie Kriterium für ein Kreis, Acta Math., 105 (1961), 245-375. MR 25:4519a

[11] J. Hass, Algorithms for recognizing knots and 3-manifolds, Chaos, Fractals and Solitons, 9 (1998), 569-581. MR 2000a:57038

[12] J. Hass, J. C. Lagarias and N. Pippenger, The computational complexity of knot and link problems, J. Assoc. Comp. Mach., 46 (1999), 185-211. MR 2000g:68056

[13] J. Hass, J. C. Lagarias and N. Pippenger, The computational complexity of knot and link problems, preliminary report, Proc. 38th Annual Symposium on Foundations of Computer Science, (1997), 172-181.

[14] J. Hass and G. P. Scott, Intersections of curves on surfaces, Israel J. Math., 51 (1985), 90-120. MR 86k:57007 
[15] J. Hempel, 3-Manifolds, Princeton University Press, Princeton, NJ, 1976. MR 54:3702

[16] J. E. Hopcroft and R. E. Tarjan, Efficient planarity testing, J. Assoc. Comp. Mach., 21 (1974), 549-568. MR 50:11841

[17] J.F.P. Hudson, Piecewise-Linear Topology, W.A. Benjamin Inc., 1969. MR 40:2094

[18] W. Jaco and H. Rubinstein, PL equivariant surgery and invariant decompositions of 3manifolds, Advances in Math., 73 (1989), 149-191. MR 90g:57016

[19] W. Jaco and J. L. Tollefson, Algorithms for the complete decomposition of a closed 3manifold, Illinois J. Math., 39 (1995), 358-406. MR 97a:57014

[20] H. Kneser, Geschlossene Flachen in dreidimensionalen Mannigfaltigkeiten, Jahresbericht Math. Verein., 28 (1929), 248-260.

[21] K. Murasugi, Knot Theory and Its Applications, Birkhauser, Boston, MA, 1996. MR 97g:57011

[22] H. Reidemeister, Knoten und Gruppen, Abh. Math. Sem., Univ. Hamburg, 5 (1926), 7-23.

[23] H. Reidemeister, Elementare Begründang der Knotentheorie, Abh. Math. Sem. Univ. Hamburg, 5 (1926), 24-32.

[24] H. Reidemeister, Knotentheorie, Springer, Berlin, 1932; Chelsea, New York, 1948. (English translation: Knot Theory, BCS Associates, Moscow, ID, 1984. MR 84j:57005)

[25] D. Rolfsen, Knots and Links, Publish or Perish Inc., Berkeley, CA, 1976. MR 58:24236

[26] C.P. Rourke and B.J. Sanderson, Introduction to Piecewise-Linear Topology, Springer-Verlag, Berlin, Heidelberg, New York, 1982. MR 83g:57009

[27] A. Schrijver, Theory of Linear and Integer Programming, John Wiley and Sons, New York, 1986. MR 88m:90090

[28] H. Schubert, Bestimmung der Primfactor zerlegung von Verkettungen, Math. Z., 76 (1961), 116-148. MR 25:4519b

[29] A. Sebö, Hilbert bases, Caratheodory's theorem and combinatorial optimization, in Integer Programming and Combinatorial Optimization, R. Kannan and W. R. Pulleybank, Eds., U. Waterloo Press, 1990, 431-455.

[30] D. J. A. Welsh, Complexity: Knots, Colourings and Counting, Cambridge University Press, Cambridge, 1993. MR 94m:57027

[31] D. J. A. Welsh, The complexity of knots, in Quo Vadis, Graph Theory?, J. Gimbel, J. Kennedy and L. V. Quintoo, eds., North-Holland, Amsterdam, 1993, 159-173. (Also: Annals Disc. Math., 55 (1993), 159-173. MR 94c:57021)

[32] D. J. A. Welsh, Knots and braids: some algorithmic questions, in Graph Structure Theory, Seattle, WA, 1991, Contemporary Math., Vol. 147, AMS, Providence, RI, 1993, 109-123. MR 94g:57014

[33] F. Y. Wu, Knot Theory and Statistical Mechanics, Rev. Mod. Phys., 64 (1992), 1099-1131. MR 94f:82025a erratum MR 94f: $82025 b$

Department of Mathematics, University of California, Davis, California 95616

E-mail address: hass@math.ucdavis.edu

Current address: School of Mathematics, Institute for Advanced Study, Princeton, New Jersey 08540

AT\&T Labs - Research, Florham Park, New Jersey 07932

E-mail address: jcl@research.att.com 